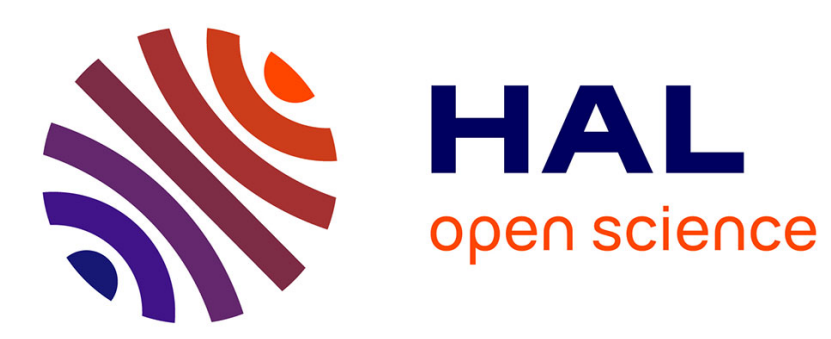

\title{
Non-negativity constraints on the pre-image for pattern recognition with kernel machines
}

Maya Kallas, Paul Honeine, Cédric Richard, Clovis Francis, Hassan Amoud

\section{To cite this version:}

Maya Kallas, Paul Honeine, Cédric Richard, Clovis Francis, Hassan Amoud. Non-negativity constraints on the pre-image for pattern recognition with kernel machines. Pattern Recognition, 2013, 46

(11), pp.3066 - 3080. 10.1016/j.patcog.2013.03.021 . hal-01965576

\section{HAL Id: hal-01965576 \\ https://hal.science/hal-01965576}

Submitted on 26 Dec 2018

HAL is a multi-disciplinary open access archive for the deposit and dissemination of scientific research documents, whether they are published or not. The documents may come from teaching and research institutions in France or abroad, or from public or private research centers.
L'archive ouverte pluridisciplinaire HAL, est destinée au dépôt et à la diffusion de documents scientifiques de niveau recherche, publiés ou non, émanant des établissements d'enseignement et de recherche français ou étrangers, des laboratoires publics ou privés. 


\title{
Non-negativity constraints on the pre-image for pattern recognition with kernel machines
}

\author{
Maya Kallas ${ }^{\mathrm{a}, \mathrm{c}}$, Paul Honeine ${ }^{\mathrm{a}, *}$, Cédric Richard ${ }^{\mathrm{b}}$, Clovis Francis $^{\mathrm{c}}$, Hassan Amoud ${ }^{\mathrm{d}}$ \\ ${ }^{a}$ Institut Charles Delaunay (CNRS), LM2S, Université de technologie de Troyes, Troyes, France \\ ${ }^{b}$ Laboratoire H. Fizeau (CNRS, OCA), Université de Nice Sophia-Antipolis, Nice, France \\ ${ }^{c}$ Laboratoire d'analyse des systèmes (LASYS), Faculté de Génie 1, Université Libanaise, Liban \\ ${ }^{d}$ Azm Center for Research in Biotechnology and its Applications, Doctoral School, Lebanese University, Lebanon
}

\section{Abstract}

Rules of physics in many real-life problems force some constraints to be satisfied. This paper deals with nonlinear pattern recognition under non-negativity constraints. While kernel principal component analysis can be applied for feature extraction or data denoising, in a feature space associated to the considered kernel function, a pre-image technique is required to go back to the input space, e.g., representing a feature in the space of input signals. The main purpose of this paper is to study a constrained pre-image problem with non-negativity constraints. We provide new theoretical results on the pre-image problem, including the weighted combination form of the pre-image, and demonstrate sufficient conditions for the convexity of the problem. The constrained problem is considered with the non-negativity, either on the pre-image itself or on the weights. We propose a simple iterative scheme to incorporate both constraints. A fortuitous side-effect of our method is the sparsity in the representation, a property investigated in this paper. Experiment results are conducted on artificial and real datasets, where many properties are investigated including the sparsity property, and compared to other methods from the literature. The relevance of the proposed method is demonstrated with experimentations on artificial data and on two types of real datasets in signal and image processing.

Keywords: kernel machines, machine learning, SVM, kernel PCA, pre-image problem, non-negativity constraints, nonlinear denoising, pattern recognition

\footnotetext{
This work is partly supported by the Lebanese University and the French-Lebanese research program CEDRE No. 10 SCI F15/L5.

${ }^{*}$ Corresponding author. Address: Université de technologie de Troyes, 12 rue Marie Curie, 10010, Troyes. Phone: +33 3257156 25. Fax: +33 325715649 .

Email addresses: maya.kallas@utt.fr (Maya Kallas), paul.honeine@utt.fr (Paul Honeine), cedric.richard@unice.fr (Cédric Richard), cfrancis@ul.edu.lb (Clovis Francis), hassan.amoud@gmail.com (Hassan Amoud)
} 


\section{Introduction}

Many applications in real-life problems require a constrained solution, including pattern recognition problems. For instance, denoising or deblurring a gray-level image should result into an image of the same type [1]. In unmixing signals or images, e.g., deconvolution, as well as in estimating some spectral feature, one may require the non-negativity of the extracted features [2]. This paper deals with a constrained nonlinear pattern recognition problem. Here, pattern recognition includes applications such as feature extraction and data denoising, where the well-known (kernel) principal component analysis (kernel PCA) is considered [3]. One may also consider other applications, such as dimensionality reduction or manifold learning. Nevertheless, it turns out that these applications can be regarded as either feature extraction or data denoising. Therefore, for clarity of presentation, we will only distinguish the latter cases.

It turns out that the non-negative constraint is very essential in many optimization problems [4]. This incorporates the mathematical equivalence between non-negative constrained optimization problems and non-positive ones. Only iterative methods can be used to solve general constrained optimization problems. Moreover, an iterative scheme for non-negativity can serve as the building block for more complex constrained optimization problems, such as the box-constrained optimization. Since the eighties, this was studied for signal deconvolution by Thomas in [5] and Prost et al. in [6]. In the beginning of the nineties, image deconvolution and deblurring were studied respectively by Thomas et al. in [7] and Snyder et al. in [8]. In the last decade, a general method for iterative optimization under non-negativity constraints has been investigated, initiated by Lantéri et al. [9], and more recently for online learning [10], system identification [11] and distributed regression [12]. Recently, such non-negativity has been introduced by the authors in [13] for feature extraction of Event-Related Potential signals, and in $[14,15]$ to denoise images.

Most investigations in constrained solutions for pattern recognition have been geared towards linear algorithms, such as the PCA in $[16,17,18,19]$. In the last couple of decades or so, kernel machines have been increasingly used to solve nonlinear learning problems, popularized since Vapnik's Support Vector Machines (SVM) [20]. While applied successfully to solve nonlinear classification, regression, and detection problems, it was not the case regarding pattern recognition. This is essentially due to the concept of the kernel trick, a "double-edged sword". In fact, the kernel trick provides a way to implicitly map data into some high-dimensional nonlinear feature space, which allows to construct nonlinear decision rules with essentially the same computational cost as linear ones. Nevertheless, one does not have access to most elements of the feature space, e.g., features or denoised elements computed using kernel PCA [21]. This is related to the 
fact that the implicit map derived by the kernel is non-surjective, with most elements of the feature space that do not have exact pre-images, and thus cannot be exactly represented in the input space.

The pre-image problem consists of mapping the pattern back from the feature space to the input space. Although the exact pre-image may seldom exists, an approximate solution is constructed. To this end, many methods have been presented in literature, starting with a fixed-point iterative algorithm proposed by Mika et al. in [22]. However, this technique was shown to be unstable, and suffers from local minima. In [23], the authors presented a pre-image technique based on a relationship between distances in both input and feature space, using Multi-Dimensional Scaling. More recently, a regularized pre-image estimation with kernel PCA is introduced in [24]. Honeine et al. in [25, 26] proposed a more direct method using relationship between inner products. See [27] for a recent review of the pre-image estimation problem. However, none of the aforementioned methods provides constrained solutions.

This paper deals with two types of non-negativity constraints, by providing a unified framework. On the first hand, the non-negativity is applied to the pre-image, and on the other hand, it is considered regarding the weights in the model. In fact, the preimage can be written as a weighted combination of the training data and thus the weights can be estimated under some constraints. A first attempt to constrain the weights is given in [28] where a penalized problem is considered with a Laplacian penalty, yielding a computationally expensive problem. In a general setting, the linear combination includes both positive and negative weights. Therefore, such weights represent contributions, without any restrictions on the signs. However, many applications cannot be interpreted by including subtracted parts within the model. This is motivated by the rules of physics, with models involving purely additive components, as illustrated for instance in $[16,17]$ for the PCA.

One of the useful properties of constraining the weights of the model is the sparsity property. In fact, the unconstrained solution can combine additive and subtractive contributions, a large part of them neutralizing others in the linear combination. By setting non-negativity constraints to these weights, it turns out that such a balance will lead to a large number of inactive components, i.e., weights close to zero. This is the property of sparsity, contributing to the widespread of Support Vector Machines algorithms [20] and compressed sensing literature [29]. We emphasize on the fact that this is a fortuitous side-effect of the non-negativity constraints, as opposed to a main sparsity objective function, where one controls the degree of sparsity of the solution. It is worth noting that including explicitly the sparsity constraint, such as minimizing an $\ell_{0}$ or an $\ell_{1}$ cost function, is computationally expensive (see for instance [18] and references therein). 
In this paper, we study a constrained solution to the pre-image problem, for nonlinear pattern recognition. To the best of our knowledge, pre-image techniques have only been applied for denoising purpose. We propose a unified framework to solve the pre-image problem for both feature extraction and denoising. We provide new theoretical results on the pre-image problem, including the weighted combination form, and provide sufficient conditions for the convexity of the problem. The constrained problem is considered with the nonnegativity, either on the pre-image or on the weights. We propose a simple iterative scheme to address both constraints, with expressions for a wide range of kernel functions. Experiment results are conducted on artificial and real datasets, where many properties are investigated including the sparsity property, and compared to other methods from the literature.

The rest of the paper is organized as follows. In the next section, we present the main idea behind kernel machines, and describe the kernel PCA technique where a unified framework for pattern recognition is proposed. Section III describes the pre-image problem and provides new theoretical results. In Section IV, we solve the pre-image problem under non-negativity constraints, either on the pre-image or on the weights. Finally, section $\mathrm{V}$ gives experimental results illustrating the efficiency of the proposed method on both artificial and real datasets.

\section{Kernel machines and kernel PCA for pattern recognition}

In recent years, kernel methods have been progressively more used due, on the one hand to the development of the statistical learning theory, and on the other hand to the computational efficiency of the corresponding algorithms. This is illustrated here with the kernel PCA, the nonlinear version of the principal component analysis.

\subsection{Kernel machines}

Let $\mathcal{X} \subset \mathbb{R}^{d}$ be an input space with the canonical (Euclidean) dot product $\boldsymbol{x}_{i} \cdot \boldsymbol{x}_{j}$ for any $\boldsymbol{x}_{i}, \boldsymbol{x}_{j} \in \mathcal{X}$. Let $\kappa: \mathcal{X} \times \mathcal{X} \mapsto \mathbb{R}$ be a symmetric and continuous function, i.e., a kernel. A kernel is positive definite if and only if any matrix $\boldsymbol{K}$ with entries $\kappa\left(\boldsymbol{x}_{i}, \boldsymbol{x}_{j}\right)$ for any finite subset of $\mathcal{X}$ is positive definite, that is $\sum_{i, j} \alpha_{i} \alpha_{j} \kappa\left(\boldsymbol{x}_{i}, \boldsymbol{x}_{j}\right) \geq 0$ for all $\alpha_{i}, \alpha_{j} \in \mathbb{R}$ and all $\boldsymbol{x}_{i}, \boldsymbol{x}_{j} \in \mathcal{X}$. Based on the Moore-Aronszajn theorem [30], any positive definite kernel guarantees the existence of a unique ${ }^{1}$ feature space (or reproducing kernel Hilbert space) $\mathcal{H}$ where $\kappa$ defines an inner product. In other words, there exists a map $\Phi: \mathcal{X} \mapsto \mathcal{H}$, from the input space to the feature space, such that

\footnotetext{
${ }^{1}$ Unique, up to an isometry.
} 


$$
\kappa\left(\boldsymbol{x}_{i}, \boldsymbol{x}_{j}\right)=\left\langle\Phi\left(\boldsymbol{x}_{i}\right), \Phi\left(\boldsymbol{x}_{j}\right)\right\rangle_{\mathcal{H}}
$$

for any $\boldsymbol{x}_{i}, \boldsymbol{x}_{j} \in \mathcal{X}$, where $\langle\cdot, \cdot\rangle_{\mathcal{H}}$ denotes the corresponding inner product in $\mathcal{H}$.

Therefore, the positive definite kernel, henceforth called (reproducing) kernel, corresponds to a generalization of the canonical dot product, and thus is a nonlinear measure of similarity between data. It turns out that most linear data processing algorithms can be easily recast in terms of dot product in input space. Substituting the dot product with a kernel offers nonlinear extensions of classical algorithms. This is referred to as the kernel trick, and can be done without the need to explicitly compute the map $\Phi$. Table 1 summarizes the most commonly used kernel functions, grouped into two classes: projective kernels, of the form

$$
\kappa\left(\boldsymbol{x}_{i}, \boldsymbol{x}_{j}\right)=f\left(\boldsymbol{x}_{i} \cdot \boldsymbol{x}_{j}\right)
$$

and radial kernels, of the form

$$
\kappa\left(\boldsymbol{x}_{i}, \boldsymbol{x}_{j}\right)=g\left(\left\|\boldsymbol{x}_{i}-\boldsymbol{x}_{j}\right\|^{2}\right)
$$

It is worth noting that some kernels induce infinite-dimensional feature spaces, such as the Gaussian kernel.

The following two propositions will be considered in this paper to demonstrate new results, and are included here for completeness. Let $f^{(k)}(\zeta)$ be the $k$-th derivative of the function $f$ with respect to $\zeta$. The following result is due to [31] (see also [32, Proposition 7.2]).

Proposition 1 (Radial kernels). A sufficient condition for a function of the form $\kappa\left(\boldsymbol{x}_{i}, \boldsymbol{x}_{j}\right)=g\left(\left\|\boldsymbol{x}_{i}-\boldsymbol{x}_{j}\right\|^{2}\right)$ to be a positive definite kernel is its complete monotonicity, i.e., its derivatives satisfies

$$
(-1)^{k} g^{(k)}(\zeta) \geq 0
$$

for any $\zeta>0$ and $k \geq 0$.

This is the case of the Gaussian kernel $\kappa_{G}\left(\boldsymbol{x}_{i}, \boldsymbol{x}_{j}\right)=g\left(\left\|\boldsymbol{x}_{i}-\boldsymbol{x}_{j}\right\|^{2}\right)$ with

$$
g^{(k)}(\zeta)=\left(-\frac{1}{2 \sigma^{2}}\right)^{k} g(\zeta)
$$

17 For the projective kernels, the following result is given in [32, Proposition 7.1]

Proposition 2 (Projective kernels). Three necessary conditions for a function $\kappa\left(\boldsymbol{x}_{i}, \boldsymbol{x}_{j}\right)=f\left(\boldsymbol{x}_{i} \cdot \boldsymbol{x}_{j}\right)$ to be a positive definite kernel are, for any non-negative $\zeta$ : 
Table 1: Commonly used reproducing kernels in machine learning, with parameters $c, \sigma>0$, and $p \in \mathbb{N}_{+}$

\begin{tabular}{|c|c|c|}
\hline & Type & General form \\
\hline 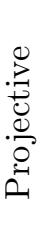 & $\begin{array}{l}\text { Monomial } \\
\text { Polynomial } \\
\text { Exponential } \\
\text { Sigmoid }\end{array}$ & $\begin{array}{c}\kappa_{m}\left(\boldsymbol{x}_{i}, \boldsymbol{x}_{j}\right)=\left(\boldsymbol{x}_{i} \cdot \boldsymbol{x}_{j}\right)^{p} \\
\kappa_{p}\left(\boldsymbol{x}_{i}, \boldsymbol{x}_{j}\right)=\left(c+\boldsymbol{x}_{i} \cdot \boldsymbol{x}_{j}\right)^{p} \\
\kappa_{E}\left(\boldsymbol{x}_{i}, \boldsymbol{x}_{j}\right)=\exp \left(\frac{1}{\sigma}\left(\boldsymbol{x}_{i} \cdot \boldsymbol{x}_{j}\right)\right) \\
\kappa_{S}\left(\boldsymbol{x}_{i}, \boldsymbol{x}_{j}\right)=\tanh \left(c\left(\boldsymbol{x}_{i} \cdot \boldsymbol{x}_{j}\right)+\sigma\right)\end{array}$ \\
\hline 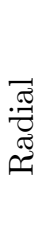 & $\begin{array}{l}\text { Laplacian } \\
\text { Gaussian } \\
\text { Multiquadratic } \\
\text { Rational }\end{array}$ & $\begin{array}{c}\kappa_{L}\left(\boldsymbol{x}_{i}, \boldsymbol{x}_{j}\right)=\exp \left(\frac{-1}{\sigma}\left\|\boldsymbol{x}_{i}-\boldsymbol{x}_{j}\right\|\right) \\
\kappa_{G}\left(\boldsymbol{x}_{i}, \boldsymbol{x}_{j}\right)=\exp \left(\frac{-1}{2 \sigma^{2}}\left\|\boldsymbol{x}_{i}-\boldsymbol{x}_{j}\right\|^{2}\right) \\
\kappa_{M Q}\left(\boldsymbol{x}_{i}, \boldsymbol{x}_{j}\right)=\sqrt{\left\|\boldsymbol{x}_{i}-\boldsymbol{x}_{j}\right\|^{2}+c} \\
\kappa_{R}\left(\boldsymbol{x}_{i}, \boldsymbol{x}_{j}\right)=1-\frac{\left\|\boldsymbol{x}_{i}-\boldsymbol{x}_{j}\right\|^{2}}{\left\|\boldsymbol{x}_{i}-\boldsymbol{x}_{j}\right\|^{2}+\sigma}\end{array}$ \\
\hline
\end{tabular}

$$
\begin{gathered}
f(\zeta) \geq 0 \\
f^{(1)}(\zeta) \geq 0 \\
f^{(1)}(\zeta)+\zeta f^{(2)}(\zeta) \geq 0
\end{gathered}
$$

\subsection{Kernel PCA}

The principal component analysis (PCA) is a powerful mathematical tool to reveal patterns within a set of data. It is a non-parametric approach, which does not incorporate any prior knowledge of the model, except its linearity. The PCA considers the most relevant eigenvectors of the data covariance matrix, i.e., eigenvectors associated to the largest eigenvalues. These eigenvectors constitute a set of orthnormal axes capturing most of the variance within data. Let us consider a set of $n$ (column-wise) data $\left\{\boldsymbol{x}_{1}, \boldsymbol{x}_{2}, \ldots, \boldsymbol{x}_{n} \in\right.$ $\mathcal{X}$ \}. Then, consider the eigen-problem $\lambda_{k} \boldsymbol{v}_{k}=\boldsymbol{C} \boldsymbol{v}_{k}$, where $\boldsymbol{C}=\frac{1}{n} \sum_{j=1}^{n} \boldsymbol{x}_{j} \boldsymbol{x}_{j}^{T}$ is the covariance matrix, data assumed to be centered around the origin. Then the $m$ eigenvectors $\left\{\boldsymbol{v}_{1}, \boldsymbol{v}_{2}, \ldots, \boldsymbol{v}_{m} \in \mathcal{X}\right\}$ are chosen from the largest eigenvalues $\lambda_{1}, \lambda_{2}, \ldots, \lambda_{m}$, where each $\lambda_{k}$ gives the amount of captured variance in the direction of $\boldsymbol{v}_{k}$. Due to the linearity of the operations, each eigenvector lies in the span of the data.

The conventional PCA identify only linear structures in a given dataset. A more generalized technique has been introduced to learn the nonlinearities using kernels, the so-called kernel PCA. The kernel PCA can reveal nonlinear kernel principal components that are more appropriate to complex and nonlinear data such as face images, handwritten digits and natural signals. For this purpose, data are (implicitly) mapped into a feature space, where PCA is applied. Although the resulting eigenvectors are linear in the feature space, they describe nonlinear relations in the input space. In order to solve this nonlinear problem, it is 
more likely to apply the kernel trick, and not to explicitly compute the map from the input to the feature space. The concept of kernel trick is illustrated here for kernel PCA [33, 34].

To this end, the PCA algorithm is recast in terms of inner product of data in feature space. Let $\Phi: \mathcal{X} \mapsto \mathcal{H}$ be a nonlinear map, and $\left\{\Phi\left(\boldsymbol{x}_{1}\right), \Phi\left(\boldsymbol{x}_{2}\right), \ldots, \Phi\left(\boldsymbol{x}_{n}\right) \in \mathcal{H}\right\}$ the set of mapped data. We wish to solve the (kernel) PCA, in terms of inner products in the feature space, $\left\langle\Phi\left(\boldsymbol{x}_{i}\right), \Phi\left(\boldsymbol{x}_{j}\right)\right\rangle_{\mathcal{H}}$, for $i, j=1,2, \ldots, n$. The covariance matrix ${ }^{2}$ in $\mathcal{H}$ is $\boldsymbol{C}^{\Phi}=\frac{1}{n} \sum_{j=1}^{n} \Phi\left(\boldsymbol{x}_{j}\right) \Phi\left(\boldsymbol{x}_{j}\right)^{T}$. The principal axes, $\varphi_{k} \in \mathcal{H}$ for $k=1,2, \ldots, m$ correspond to the eigenvectors with the largest eigenvalues $\lambda_{k}$ satisfying the expression

$$
\lambda_{k} \varphi_{k}=\boldsymbol{C}^{\Phi} \varphi_{k}
$$

By analogy with the classical PCA, any solution $\varphi_{k}$ lies in the span of the $\Phi$-images of the data. This implies that there exist some coefficients $\alpha_{1}, \alpha_{2}, \ldots, \alpha_{n}$ such that

$$
\varphi_{k}=\sum_{i=1}^{n} \alpha_{k, i} \Phi\left(\boldsymbol{x}_{i}\right) .
$$

This is a more general result known as the representer theorem $[35,36]$ in kernel machines, where the solution of a (regularized) learning problem can be written in terms of a linear combination of the training data in the feature space.

Replacing the expression of $\boldsymbol{C}^{\Phi}$ and the representer (5) into the eigen-problem (4), we get the new eigen-problem in terms of inner product with

$$
n \lambda_{k} \boldsymbol{\alpha}_{k}=\boldsymbol{K} \boldsymbol{\alpha}_{k}
$$

where $\boldsymbol{K}$ is the $n \times n$ matrix of entries $\kappa\left(\boldsymbol{x}_{i}, \boldsymbol{x}_{j}\right)$ with (1) applied, and $\boldsymbol{\alpha}_{k}=\left[\begin{array}{llll}\alpha_{k, 1} & \alpha_{k, 2} & \cdots & \alpha_{k, n}\end{array}\right]^{T}$. Furthermore, two issues are considered in the final kernel PCA algorithm. First, as mentioned earlier, data should be centered in the feature space. This can be done by substituting the matrix $\boldsymbol{K}$ by $\left(\mathbf{I}-\mathbf{1}_{n}\right) \boldsymbol{K}\left(\mathbf{I}-\mathbf{1}_{n}\right)$, where $\mathbf{I}$ is the identity matrix and $\mathbf{1}_{n}$ is a $n \times n$ matrix of entries $1 / n$. Second, we normalize as in PCA by requiring that the corresponding vectors in $\mathcal{H}$ be unit-norm, i.e., $\left\langle\varphi_{k}, \varphi_{k}\right\rangle_{\mathcal{H}}=1$. This is done by rescaling the weight vectors $\boldsymbol{\alpha}_{k}$ such that $\lambda_{k}\left(\boldsymbol{\alpha}_{k} \cdot \boldsymbol{\alpha}_{k}\right)=1$, for $k=1,2, \ldots, m$.

\footnotetext{
${ }^{2}$ We assume that data are centered in $\mathcal{H}$; otherwise we apply the algorithm by substituting each $\Phi\left(\boldsymbol{x}_{j}\right)$ with $\Phi\left(\boldsymbol{x}_{j}\right)-$ $\frac{1}{n} \sum_{i=1}^{n} \Phi\left(\boldsymbol{x}_{i}\right)$.
} 


\subsection{Kernel PCA for pattern recognition}

Roughly speaking, two main applications can be given with conventional PCA: Either consider relevant principal axes as extracted features, or project some noisy observation onto (the subspace spanned by) these axes as a denoising scheme. Both techniques are illustrated here in the feature space, using kernel PCA.

\subsubsection{Feature extraction}

Kernel-PCA defines the set of most relevant axes in the feature space. Let $\left\{\varphi_{1}, \varphi_{2}, \ldots, \varphi_{m} \in \mathcal{H}\right\}$ be the set of these axes. Then each $\varphi_{k}$ takes the form (5), namely

$$
\varphi_{k}=\sum_{j=1}^{n} \alpha_{k, j} \Phi\left(\boldsymbol{x}_{j}\right)
$$

where $\alpha_{k, 1}, \alpha_{k, 2}, \ldots, \alpha_{k, n}$ are obtained from the eigenvector associated to the $k$-th eigenvalue in (6). We also define the relevant subspace of $\mathcal{H}$ as the one spanned by these axes. By analogy to the conventional PCA, these axes (as well as the associated subspace) capture most of the variance of the data. They can be regarded as features extracted from the data, capturing the largest variations and orthonormal to each others.

\subsubsection{Denoising}

Denoising is a technique applied in order to recognize patterns corrupted by noise. Let $\boldsymbol{x}_{0} \in \mathcal{X}$ be a noisy sample. Then the associated image $\Phi\left(\boldsymbol{x}_{0}\right)$ is projected onto the relevant subspace (described above), resulting into the denoised pattern. The latter is expressed by the inner product of the mapped sample with the $m$ principal axes, as

$$
\varphi=\sum_{k=1}^{m}\left\langle\Phi\left(\boldsymbol{x}_{0}\right), \varphi_{k}\right\rangle_{\mathcal{H}} \varphi_{k} .
$$

Expanding this expression by (5) and applying the equivalence between the inner product operator and the kernel function $\kappa$, we get

$$
\begin{aligned}
\varphi & =\sum_{k=1}^{m}\left\langle\Phi\left(\boldsymbol{x}_{0}\right), \sum_{i=1}^{n} \alpha_{k, i} \Phi\left(\boldsymbol{x}_{i}\right)\right\rangle_{\mathcal{H}} \sum_{j=1}^{n} \alpha_{k, j} \Phi\left(\boldsymbol{x}_{j}\right) \\
& =\sum_{k=1}^{m} \sum_{i=1}^{n} \alpha_{k, i} \kappa\left(\boldsymbol{x}_{0}, \boldsymbol{x}_{i}\right) \sum_{j=1}^{n} \alpha_{k, j} \Phi\left(\boldsymbol{x}_{j}\right)
\end{aligned}
$$


Table 2: Unified view for the definition of $\gamma_{j}$ in $\varphi=\sum_{j=1}^{n} \gamma_{j} \Phi\left(\boldsymbol{x}_{j}\right)$

\begin{tabular}{lc}
\multicolumn{1}{c}{ Application } & $\gamma_{j}$ \\
\hline \hline Feature extraction of $\varphi_{k}$ & $\alpha_{k, j}$ \\
Denoising of $\boldsymbol{x}_{0}$ & $\sum_{k=1}^{m} \sum_{i=1}^{n} \alpha_{k, i} \alpha_{k, j} \kappa\left(\boldsymbol{x}_{0}, \boldsymbol{x}_{i}\right)$
\end{tabular}

\subsubsection{A unifying view}

Now, we propose a unified view to tackle both above pattern recognition problems. To this end, we write the extracted feature and the denoised pattern as a linear combination of the mapped training data, with $\varphi_{k}=\sum_{j=1}^{n} \alpha_{k, j} \Phi\left(\boldsymbol{x}_{j}\right)$ and $\varphi=\sum_{j=1}^{n}\left[\sum_{k=1}^{m} \sum_{i=1}^{n} \alpha_{k, i} \alpha_{k, j} \kappa\left(\boldsymbol{x}_{0}, \boldsymbol{x}_{i}\right)\right] \Phi\left(\boldsymbol{x}_{j}\right)$. Aggregating all these terms, we get a unifying view of both cases, with

$$
\varphi=\sum_{j=1}^{n} \gamma_{j} \Phi\left(\boldsymbol{x}_{j}\right)
$$

On the one hand, the feature extraction is given as $\varphi=\varphi_{k}$ with

$$
\gamma_{j}=\alpha_{k, j}
$$

and on the other hand, the denoising pattern with

$$
\gamma_{j}=\sum_{k=1}^{m} \sum_{i=1}^{n} \alpha_{k, i} \alpha_{k, j} \kappa\left(\boldsymbol{x}_{0}, \boldsymbol{x}_{i}\right) .
$$

In the latter case, the coefficients $\gamma_{j}$ depend on the noisy $\boldsymbol{x}_{0}$, which can be either a new observation or one of the training data. Summarized in Table 2, the unifying expression in (7) enables us to define a general form in the optimization problem for both feature extraction and denoising schemes.

\section{The pre-image problem}

Classically, the kernel PCA has shown its powerful ability in supervised learning, as a pre-processing stage followed by a discrimination rule. In these cases, for any given $\boldsymbol{x}_{0}$, the projection of $\Phi\left(\boldsymbol{x}_{0}\right)$ onto any $\varphi \in \mathcal{H}$ of the form (7), can be defined by $\left\langle\varphi, \Phi\left(\boldsymbol{x}_{0}\right)\right\rangle_{\mathcal{H}}=\sum_{j=1}^{n} \gamma_{j} \kappa\left(\boldsymbol{x}_{j}, \boldsymbol{x}_{0}\right)$, and comparing it to a threshold gives the decision rule. The problem can be easily solved, with the coefficients computed using the kernel trick. However, in pattern recognition such as feature extraction and denoising, we are interested in the 


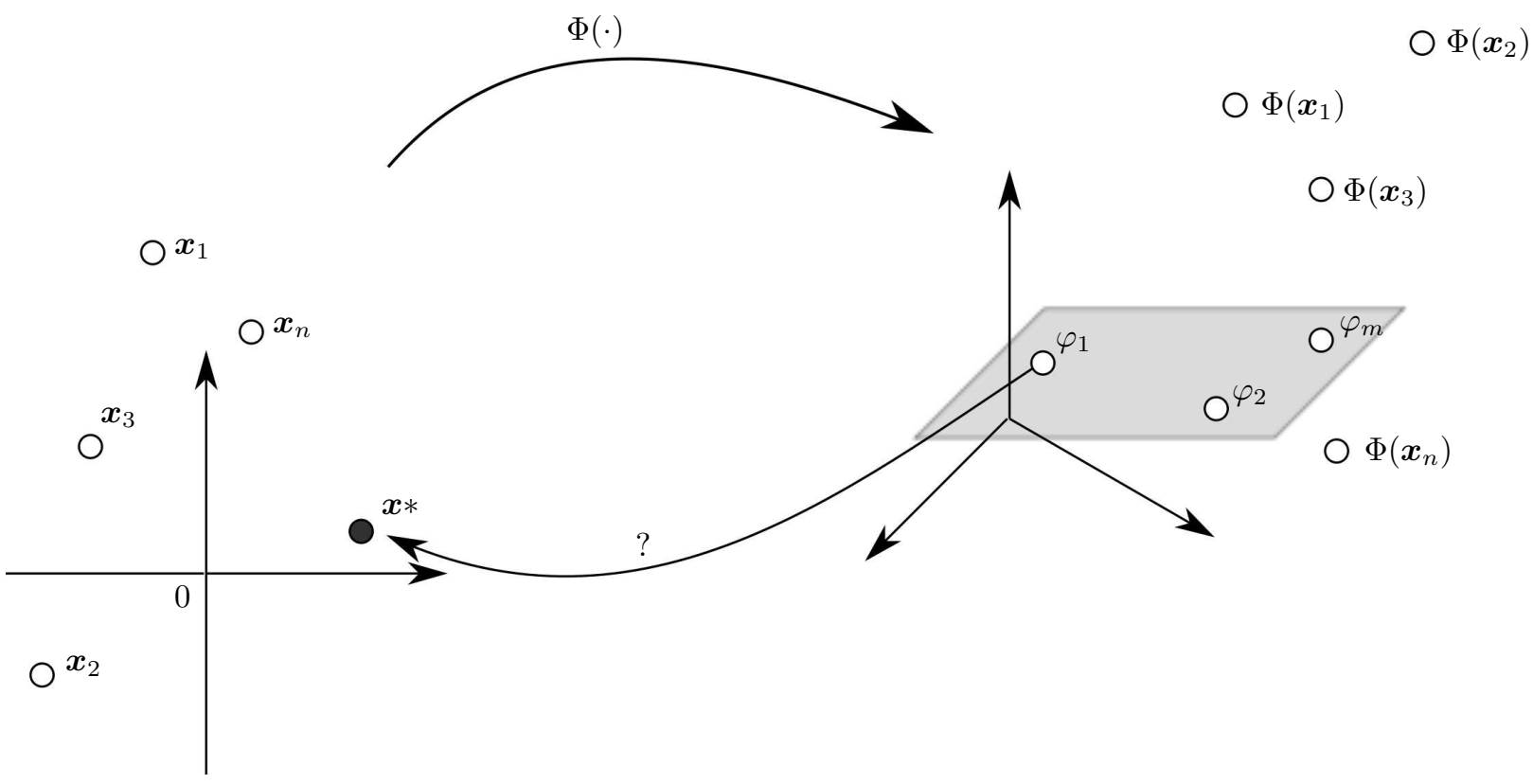

Figure 1: Schematic illustration of the pre-image problem. Constructed in the feature space from some training data, principal axes are mapped back to the input space by solving the pre-image problem, pre-imaging $\varphi_{1}$ into $\boldsymbol{x}^{*}$ here.

feature itself. More likely, we seek its counterpart in the input space, the observation space. It is natural to have extracted patterns of the same type as the data, i.e., identical input space, since one often seeks for a signal as a pattern in signal processing, or a denoised image in image processing. The pre-image problem is illustrated in Figure 1.

With the exception of the $\Phi$-images of the training data, only a very few elements in the feature space have pre-images, i.e., data which maps into (7) for some given coefficients. In fact, this is an ill-posed problem since the exact pre-image may not exist, and even if it exists, it might be not unique. To solve this problem, we seek an approximate solution, i.e., a $\boldsymbol{x}^{*} \in \mathcal{X}$ whose image $\Phi\left(\boldsymbol{x}^{*}\right)$ is as close as possible to $\varphi$. The way back from the feature space to the input space is called the pre-image problem. Initially studied by Mika et al. in [22] for denoising purpose, it consists of solving the optimization problem

$$
\boldsymbol{x}^{*}=\arg \min _{\boldsymbol{x} \in \mathcal{X}} \frac{1}{2}\|\varphi-\Phi(\boldsymbol{x})\|_{\mathcal{H}}^{2},
$$

where $\|\cdot\|_{\mathcal{H}}$ denotes the norm in $\mathcal{H}$, and thus provides a measure of distance between elements in the feature space, with the norm of their residue. Thanks to the unifying view given in (7) with $\varphi=\sum_{i=1}^{n} \gamma_{i} \Phi\left(\boldsymbol{x}_{i}\right)$, we consider the same optimization problem for either feature extraction or denoising, with 
Table 3: Gradient of the cost function (9) for most commonly used kernels, with respect to either $\boldsymbol{x}$ (second column) or $\boldsymbol{\beta}$ from $\boldsymbol{x}=\boldsymbol{\beta}^{T} \boldsymbol{X}$ (third column).

$\begin{array}{ll}\text { Type } & \nabla_{\boldsymbol{x}} J(\boldsymbol{x}) \quad \nabla_{\boldsymbol{\beta}} J\left(\boldsymbol{\beta}^{T} \boldsymbol{X}\right)\end{array}$

\begin{tabular}{lcc}
\hline \hline Polynomial & $-\sum_{i=1}^{n} \gamma_{i} p \kappa_{p-1}\left(\boldsymbol{x}_{i}, \boldsymbol{x}\right) \boldsymbol{x}_{i}+p \kappa_{p-1}(\boldsymbol{x}, \boldsymbol{x}) \boldsymbol{x}$ & $-\sum_{i=1}^{n} \gamma_{i} p \kappa_{p-1}\left(\boldsymbol{x}_{i}, \boldsymbol{\beta}^{T} \boldsymbol{X}\right) \boldsymbol{x}_{i} \boldsymbol{X}^{T}+p \kappa_{p-1}\left(\boldsymbol{\beta}^{T} \boldsymbol{X}, \boldsymbol{\beta}^{T} \boldsymbol{X}\right) \boldsymbol{\beta}^{T} \boldsymbol{X} \boldsymbol{X}^{T}$ \\
Sigmoid & $-\sum_{i=1}^{n} \gamma_{i}\left(1-\kappa_{S}^{2}\left(\boldsymbol{x}_{i}, \boldsymbol{x}\right)\right) c \boldsymbol{x}_{i}+c\left(1-\kappa_{S}^{2}(\boldsymbol{x}, \boldsymbol{x})\right) \boldsymbol{x}$ & $-\sum_{i=1}^{n} \gamma_{i} c\left(1-\kappa_{S}^{2}\left(\boldsymbol{x}_{i}, \boldsymbol{\beta}^{T} \boldsymbol{X}\right)\right) \boldsymbol{x}_{i} \boldsymbol{X}^{T}+c\left(1-\kappa_{S}^{2}\left(\boldsymbol{\beta}^{T} \boldsymbol{X}, \boldsymbol{\beta}^{T} \boldsymbol{X}\right)\right) \boldsymbol{\beta}^{T} \boldsymbol{X} \boldsymbol{X}^{T}$ \\
Exponential & $-\frac{1}{\sigma} \sum_{i=1}^{n} \gamma_{i} \kappa_{E}\left(\boldsymbol{x}_{i}, \boldsymbol{x}\right) \boldsymbol{x}_{i}+\frac{1}{\sigma} \kappa_{E}(\boldsymbol{x}, \boldsymbol{x}) \boldsymbol{x}$ & $-\frac{1}{\sigma} \sum_{i=1}^{n} \gamma_{i} \kappa_{E}\left(\boldsymbol{x}_{i}, \boldsymbol{\beta}^{T} \boldsymbol{X}\right) \boldsymbol{x}_{i} \boldsymbol{X}^{T}+\frac{1}{\sigma} \kappa_{E}\left(\boldsymbol{\beta}^{T} \boldsymbol{X}, \boldsymbol{\beta}^{T} \boldsymbol{X}\right) \boldsymbol{\beta}^{T} \boldsymbol{X} \boldsymbol{X}^{T}$ \\
Gaussian & $-\frac{1}{\sigma^{2}} \sum_{i=1}^{n} \gamma_{i} \kappa_{G}\left(\boldsymbol{x}_{i}, \boldsymbol{x}\right)\left(\boldsymbol{x}_{i}-\boldsymbol{x}\right)$ & $-\frac{1}{\sigma^{2}} \sum_{i=1}^{n} \gamma_{i} \kappa_{G}\left(\boldsymbol{x}_{i}, \boldsymbol{\beta}^{T} \boldsymbol{X}\right)\left(\boldsymbol{x}_{i}-\boldsymbol{\beta}^{T} \boldsymbol{X}\right) \boldsymbol{X}^{T}$
\end{tabular}

$$
\boldsymbol{x}^{*}=\arg \min _{\boldsymbol{x} \in \mathcal{X}} \frac{1}{2}\left\|\sum_{i=1}^{n} \gamma_{i} \Phi\left(\boldsymbol{x}_{i}\right)-\Phi(\boldsymbol{x})\right\|_{\mathcal{H}}^{2}
$$

The general form used for the calculation is described by

$$
\boldsymbol{x}^{*}=\arg \min _{\boldsymbol{x}} J(\boldsymbol{x})
$$

where $J(\boldsymbol{x})$ represents the resulting cost function, defined by

$$
J(\boldsymbol{x})=-\sum_{i=1}^{n} \gamma_{i} \kappa\left(\boldsymbol{x}_{i}, \boldsymbol{x}\right)+\frac{1}{2} \kappa(\boldsymbol{x}, \boldsymbol{x})
$$

with $\gamma_{j}$ given in Table 2 . In this expression, the term $\frac{1}{2} \sum_{i=1}^{n} \sum_{j=1}^{n} \gamma_{i} \gamma_{j} \kappa\left(\boldsymbol{x}_{i}, \boldsymbol{x}_{j}\right)$ has been removed since it is independent of $\boldsymbol{x}$.

This is a highly nonlinear optimization problem. To solve this problem, one may study the gradient of the cost function $J(\boldsymbol{x})$ with respect to $\boldsymbol{x}$. At an optimum, the gradient with respect to $\boldsymbol{x}$ disappears, namely $\nabla_{\boldsymbol{x}} J(\boldsymbol{x})=0$. The resulting gradient is given as

$$
\nabla_{\boldsymbol{x}} J(\boldsymbol{x})=-\sum_{i=1}^{n} \gamma_{i} \frac{\partial \kappa\left(\boldsymbol{x}_{i}, \boldsymbol{x}\right)}{\partial \boldsymbol{x}}+\frac{1}{2} \frac{\partial \kappa(\boldsymbol{x}, \boldsymbol{x})}{\partial \boldsymbol{x}} .
$$

This is the general form for all kernels, including for instance the projective kernels of the form (2) such as the polynomial kernel. Expressions (9)-(10) can be further simplified for the wide class of radial kernels, of the form (3) such as the Gaussian kernel. In such cases, $\kappa(\boldsymbol{x}, \boldsymbol{x})$ is independent of $\boldsymbol{x}$, for all $\boldsymbol{x} \in \mathcal{X}$, thus $\partial \kappa(\boldsymbol{x}, \boldsymbol{x}) / \partial \boldsymbol{x}$ equals to zero, and only the first term of (10) remains. See Table 1 for expressions of commonly used kernels. 
The final result of (12) can thus be expressed as

$$
\boldsymbol{x}^{*}=\sum_{i=1}^{n} \gamma_{i} \frac{g^{(1)}\left(\left\|\boldsymbol{x}_{i}-\boldsymbol{x}^{*}\right\|^{2}\right)}{\sum_{j=1}^{n} \gamma_{j} g^{(1)}\left(\left\|\boldsymbol{x}_{j}-\boldsymbol{x}^{*}\right\|^{2}\right)} \boldsymbol{x}_{i},
$$

232 again of the form $\boldsymbol{x}^{*}=\sum_{i=1}^{n} \beta_{i}^{*} \boldsymbol{x}_{i}$.

$$
\boldsymbol{x}^{*}=\sum_{i=1}^{n} \beta_{i}^{*} \boldsymbol{x}_{i}
$$

where $\beta_{i}^{*}$ are weights to be determined.

$$
\sum_{i=1}^{n} \gamma_{i} \frac{\partial \kappa\left(\boldsymbol{x}_{i}, \boldsymbol{x}^{*}\right)}{\partial \boldsymbol{x}^{*}}=\frac{1}{2} \frac{\partial \kappa\left(\boldsymbol{x}^{*}, \boldsymbol{x}^{*}\right)}{\partial \boldsymbol{x}^{*}} .
$$
be written as

$$
\sum_{i=1}^{n} \gamma_{i} \frac{\partial \kappa\left(\boldsymbol{x}_{i}, \boldsymbol{x}^{*}\right)}{\partial \boldsymbol{x}^{*}}=\sum_{i=1}^{n} \gamma_{i} \frac{\partial f\left(\boldsymbol{x}_{i} \cdot \boldsymbol{x}^{*}\right)}{\partial\left(\boldsymbol{x}_{i} \cdot \boldsymbol{x}^{*}\right)} \boldsymbol{x}_{i},
$$

$$
\frac{1}{2} \frac{\partial \kappa\left(\boldsymbol{x}^{*}, \boldsymbol{x}^{*}\right)}{\partial \boldsymbol{x}^{*}}=\frac{1}{2} \frac{\partial f\left(\boldsymbol{x}^{*} \cdot \boldsymbol{x}^{*}\right)}{\partial\left(\boldsymbol{x}^{*} \cdot \boldsymbol{x}^{*}\right)} 2 \boldsymbol{x}^{*} .
$$

Combining both expressions, the equation (12) becomes

$$
\boldsymbol{x}^{*}=\sum_{i=1}^{n} \gamma_{i} \frac{f^{(1)}\left(\boldsymbol{x}_{i} \cdot \boldsymbol{x}^{*}\right)}{f^{(1)}\left(\boldsymbol{x}^{*} \cdot \boldsymbol{x}^{*}\right)} \boldsymbol{x}_{i}
$$

of the form $\boldsymbol{x}^{*}=\sum_{i=1}^{n} \beta_{i}^{*} \boldsymbol{x}_{i}$. vanishes. The gradient at the optimum, (12), can be written as

$$
\sum_{i=1}^{n} \gamma_{i} \frac{\partial \kappa\left(\boldsymbol{x}_{i}, \boldsymbol{x}^{*}\right)}{\partial \boldsymbol{x}^{*}}=0
$$

$$
\sum_{i=1}^{n} \gamma_{i} \frac{\partial \kappa\left(\boldsymbol{x}_{i}, \boldsymbol{x}^{*}\right)}{\partial \boldsymbol{x}^{*}}=\sum_{i=1}^{n} \gamma_{i} \frac{\partial g\left(\left\|\boldsymbol{x}_{i}-\boldsymbol{x}^{*}\right\|^{2}\right)}{\partial\left(\left\|\boldsymbol{x}_{i}-\boldsymbol{x}^{*}\right\|^{2}\right)} 2\left(\boldsymbol{x}^{*}-\boldsymbol{x}_{i}\right) .
$$

Proof. To prove this, we consider separately the two classes of kernels: projective and radial kernels (see Table 1). Using the expression of the gradient (10), we have at the optimum $\nabla_{\boldsymbol{x}} J\left(\boldsymbol{x}^{*}\right)=0$, namely

Let us begin with the projective kernels, of the form (2). Thus, the left-hand-side of this equation can

We now study the class of radial kernels, defined by expression (3). In such case, the term $\partial \kappa(\boldsymbol{x}, \boldsymbol{x}) / \partial \boldsymbol{x}$ 
The following result provides new insight into the connection between the weights in both feature and input spaces.

Corollary 1. When input data are non-negative, if the weights in the feature space are non-negative, i.e., $\gamma_{1}, \gamma_{2}, \ldots, \gamma_{n} \geq 0$, then the weights of the corresponding pre-image are also non-negative, i.e., $\beta_{1}^{*}, \beta_{2}^{*}, \ldots, \beta_{n}^{*} \geq 0$. Moreover, the non-negativity of the data is not required for radial kernels.

Proof. For the projective kernels, we have from (13):

$$
\beta_{i}^{*}=\gamma_{i} \frac{f^{(1)}\left(\boldsymbol{x}_{i} \cdot \boldsymbol{x}^{*}\right)}{f^{(1)}\left(\boldsymbol{x}^{*} \cdot \boldsymbol{x}^{*}\right)} .
$$

When all input data are non-negative, the above derivatives are non-negative due to Proposition 2. The same proof can be applied for the radial kernels by applying Proposition 1 to (14) with

$$
\beta_{i}^{*}=\gamma_{i} \frac{g^{(1)}\left(\left\|\boldsymbol{x}_{i}-\boldsymbol{x}^{*}\right\|^{2}\right)}{\sum_{j=1}^{n} \gamma_{j} g^{(1)}\left(\left\|\boldsymbol{x}_{j}-\boldsymbol{x}^{*}\right\|^{2}\right)} .
$$

The above results are based on the first derivative of the cost function (9). Its second derivative provides a deeper insight on its convexity, as derived in the following theorem.

Theorem 2. For the class of radial kernels, a sufficient condition for the convexity of the cost function is given by the non-negativity of the coefficients $\gamma_{1}, \gamma_{2}, \ldots, \gamma_{n}$.

Proof. Taking the second derivative of the cost function (9) with respect to $\boldsymbol{x}$, we get

$$
\begin{aligned}
\nabla_{\boldsymbol{x}}^{2} J(\boldsymbol{x}) & =\nabla_{\boldsymbol{x}}\left[2 \sum_{i=1}^{n} \gamma_{i}\left(\boldsymbol{x}_{i}-\boldsymbol{x}\right) g^{(1)}\left(\left\|\boldsymbol{x}_{i}-\boldsymbol{x}\right\|^{2}\right)\right] \\
& =2 \sum_{i=1}^{n} \gamma_{i}\left(-g^{(1)}\left(\left\|\boldsymbol{x}_{i}-\boldsymbol{x}\right\|^{2}\right)+2\left(\boldsymbol{x}_{i}-\boldsymbol{x}\right)^{2} g^{(2)}\left(\left\|\boldsymbol{x}_{i}-\boldsymbol{x}\right\|^{2}\right)\right) .
\end{aligned}
$$

The term between parentheses is positive, due to Proposition 1. Therefore, a sufficient condition for the second derivative to be non-negative, and thus for the convexity of (9), is that all the coefficients $\gamma_{i}$ are non-negative.

The non-negativity of the coefficients $\gamma_{i}$ 's is a condition imposed by the SVM for classification and regression, as well as some other machine learning methods. However, this is not the case in general, with the kernel PCA for instance. In this paper, we will not limit ourselves to the convex problem, but consider the more general non-convex problem.

From Theorem 1, the form $\boldsymbol{x}^{*}=\sum_{i=1}^{n} \beta_{i}^{*} \boldsymbol{x}_{i}$ provides a fixed-point iterative method to solve the pre-image problem, where the $\beta_{i}$ 's depend on $\boldsymbol{x}^{*}$. For the Gaussian kernel, we have

$$
\kappa_{G}\left(\boldsymbol{x}_{i}, \boldsymbol{x}_{j}\right)=g\left(\left\|\boldsymbol{x}_{i}-\boldsymbol{x}_{j}\right\|^{2}\right)=\exp \left(\frac{-1}{2 \sigma^{2}}\left\|\boldsymbol{x}_{i}-\boldsymbol{x}_{j}\right\|^{2}\right)
$$


thus

$$
g^{(1)}\left(\left\|\boldsymbol{x}_{i}-\boldsymbol{x}_{j}\right\|\right)=-\frac{1}{2 \sigma^{2}} \kappa_{G}\left(\boldsymbol{x}_{i}, \boldsymbol{x}_{j}\right)
$$

From expression (14), we get the fixed-point iterative method for the Gaussian kernel

$$
\boldsymbol{x}^{*}=\frac{\sum_{i=1}^{n} \gamma_{i} \kappa_{G}\left(\boldsymbol{x}_{i}, \boldsymbol{x}^{*}\right) \boldsymbol{x}_{i}}{\sum_{i=1}^{n} \gamma_{i} \kappa_{G}\left(\boldsymbol{x}_{i}, \boldsymbol{x}^{*}\right)} .
$$

When the polynomial kernel is applied, with

$$
\kappa_{p}\left(\boldsymbol{x}_{i}, \boldsymbol{x}_{j}\right)=f\left(\boldsymbol{x}_{i} \cdot \boldsymbol{x}_{j}\right)=\left(c+\boldsymbol{x}_{i} \cdot \boldsymbol{x}_{j}\right)^{p},
$$

then

$$
f^{(1)}\left(\boldsymbol{x}_{i} \cdot \boldsymbol{x}_{j}\right)=p \kappa_{p-1}\left(\boldsymbol{x}_{i}, \boldsymbol{x}_{j}\right),
$$

where $\kappa_{p-1}\left(\boldsymbol{x}_{i}, \boldsymbol{x}_{j}\right)=f\left(\boldsymbol{x}_{i} \cdot \boldsymbol{x}_{j}\right)=\left(c+\boldsymbol{x}_{i} \cdot \boldsymbol{x}_{j}\right)^{p-1}$. From expression (13), we get the fixed-point iterative method for the polynomial kernel

$$
\boldsymbol{x}^{*}=\frac{\sum_{i=1}^{n} \gamma_{i} \kappa_{p-1}\left(\boldsymbol{x}_{i}, \boldsymbol{x}^{*}\right) \boldsymbol{x}_{i}}{\kappa_{p-1}\left(\boldsymbol{x}^{*}, \boldsymbol{x}^{*}\right)} .
$$

Table 3 (second column) illustrates the diversity of the gradient expressions for different kernels. Such fixed-point iterative algorithm suffers from instabilities and even may not converge at all, as illustrated in [22] where only the Gaussian kernel was used. Moreover, results widely vary for different starting points in practice. These issues are likely due to two factor: First, the absence of a stepsize parameter, which allows to control the convergence of the algorithm. Second, the unconstrained solution, as the hypothesis space corresponds to the whole input space. Both issues will be addressed in next section.

\section{The pre-image under non-negativity constraints}

In many applications in pattern recognition, one seeks non-negativity in the solution. In image processing for instance, training data are images or patches within an image, i.e., data which are non-negative for graylevel images. To get a feature extracted or a denoised version of the same type (same input space with non-negativity of each pixel), one should impose non-negativity constraints on the pre-image. However, the constraints are applied either on the data itself, or on the weights model using the linear combination of (11). 


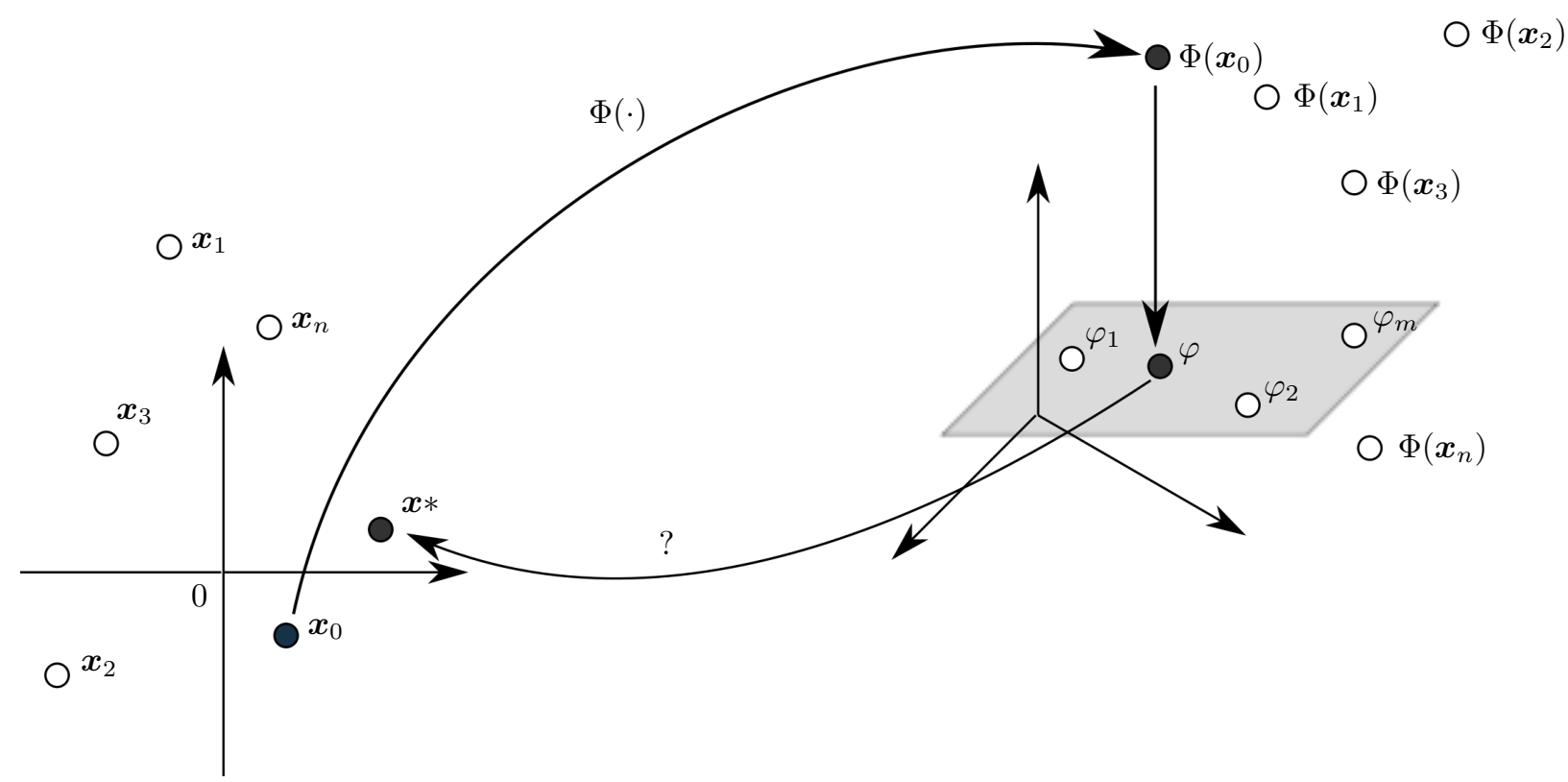

Figure 2: Schematic illustration of the pre-image problem under the non-negativity constraints. A given noisy data $\boldsymbol{x}_{0}$ is mapped into $\Phi\left(\boldsymbol{x}_{0}\right)$, then projected into the subspace spanned by the most relevant principal axes $\varphi_{1}, \varphi_{2}, \ldots, \varphi_{m}$. The denoised pattern $\varphi$ is mapped back to the input space, into $\boldsymbol{x}^{*}$.

$$
\boldsymbol{x}^{*}=\arg \min _{\boldsymbol{x}} J(\boldsymbol{x}) \quad \text { subject to } \boldsymbol{x} \geq 0
$$

where expression $\boldsymbol{x} \geq 0$ refers to the non-negativity of all entries of the vector $\boldsymbol{x}$. The gradient of $J(\cdot)$ is given in Table 3 for several kernel types. Next, we derive an iterative updating rule that leads to the non-negativity of pre-image. Figure 2 illustrate the concept of this constrained pre-image.

A general form of the pre-image problem under non-negativity constraints is defined in (16). Consider the Lagrangian function associated to this constrained optimization problem, with ${ }^{3}$

$$
J(\boldsymbol{x})-\boldsymbol{\mu}^{T} \boldsymbol{x},
$$

where $\boldsymbol{\mu}$ represents the vector of non-negative Lagrange multipliers. At the optimum solution $\boldsymbol{x}^{*}$, corresponding to the optimal multiplier vector $\boldsymbol{\mu}^{*}$, the first-order (Karush-)Kuhn-Tucker optimality conditions

\footnotetext{
${ }^{3}$ A more general form can be given using a function expressing the constraints, $g(\boldsymbol{x})$, with the Lagrangian expression $J(\boldsymbol{x})-\boldsymbol{\mu}^{T} g(\boldsymbol{x})$ [9]. For clarity of this paper, this function is substituted with its simplest form, $\boldsymbol{x}$.
} 
are satisfied, with

$$
\begin{aligned}
\nabla_{\boldsymbol{x}}\left[J\left(\boldsymbol{x}^{*}\right)-\boldsymbol{\mu}^{* T} \boldsymbol{x}^{*}\right] & =0 \\
\mu_{i}^{*} x_{i}^{*} & =0 \text { for all } i=1,2, \ldots
\end{aligned}
$$

In practice, one may use a stepsize independent of $i$, which satisfies the following inequality

$$
\eta(t) \leq \min _{i} \frac{1}{\left[\nabla_{\boldsymbol{x}} J(\boldsymbol{x}(t))\right]_{i}} .
$$

299 Written in matrix form, the final updating rule is defined by

$$
\boldsymbol{x}(t+1)=\boldsymbol{x}(t)-\eta(t) \operatorname{diag}[\boldsymbol{x}(t)] \nabla_{\boldsymbol{x}} J(\boldsymbol{x}(t)),
$$


Table 4: Values of the parameters for the three datasets.

\begin{tabular}{|lc|c|c|c|}
\cline { 3 - 5 } \multicolumn{1}{c|}{} & \multicolumn{3}{c|}{ datasets } \\
\cline { 3 - 5 } \multicolumn{1}{c|}{} & & banana & donut & frame \\
\hline Noise parameter & $\nu$ & 0.2 & 0.4 & 0.2 \\
Number of training data & $n$ & 800 & 500 & 550 \\
Number of eigenvectors & $m$ & 2 & 4 & 4 \\
Bandwidth of the Gaussian kernel & $\sigma$ & 0.7 & 0.8 & 0.5 \\
Number of denoised data & $N$ & 200 & 100 & 510 \\
Value of the stepsize parameter & $\eta$ & 0.3 & 0.3 & 0.3 \\
Number of iterations & $t_{\max }$ & 20 & 20 & 20 \\
\hline
\end{tabular}

The corresponding cost function (9) can be written as

$$
J\left(\boldsymbol{\beta}^{T} \boldsymbol{X}\right)=-\sum_{i=1}^{n} \gamma_{i} \kappa\left(\boldsymbol{x}_{i}, \boldsymbol{\beta}^{T} \boldsymbol{X}\right)+\frac{1}{2} \kappa\left(\boldsymbol{\beta}^{T} \boldsymbol{X}, \boldsymbol{\beta}^{T} \boldsymbol{X}\right) .
$$

${ }_{311}$ Taking the gradient of the above expression with respect to $\boldsymbol{\beta}$, we get

$$
\nabla_{\boldsymbol{\beta}} J\left(\boldsymbol{\beta}^{T} \boldsymbol{X}\right)=\nabla_{\boldsymbol{x}} J(\boldsymbol{x}) \boldsymbol{X}^{T},
$$


where $\boldsymbol{x}=\boldsymbol{\beta}^{T} \boldsymbol{X}$. Table 3 (third column) gives the gradient with respect to $\boldsymbol{\beta}$ of the most commonly used kernels. The relationship between these expressions and the gradient with respect to $\boldsymbol{x}$ (second column in Table 3) is given in expression (19).

By deriving this analogy with the constrained optimization problem (16) (non-negativity on the preimage), we revisit the latter in order to impose the non-negativity on the weights $\boldsymbol{\beta}^{*}$ in the expansion $\boldsymbol{x}^{*}=\boldsymbol{\beta}^{* T} \boldsymbol{X}$. This yields the following optimization problem

$$
\boldsymbol{\beta}^{*}=\arg \min _{\boldsymbol{\beta}} J\left(\boldsymbol{\beta}^{T} \boldsymbol{X}\right) \quad \text { subject to } \boldsymbol{\beta} \geq 0
$$

In this expression $J(\cdot)$ is defined as in (18), with its gradient with respect to $\boldsymbol{\beta}$ given in (19). From (17), the updating rule of these weights is given as

$$
\boldsymbol{\beta}(t+1)=\boldsymbol{\beta}(t)-\eta(t) \operatorname{diag}[\boldsymbol{\beta}(t)] \nabla_{\boldsymbol{x}} J(\boldsymbol{x}) \boldsymbol{X}^{T},
$$

where $\boldsymbol{x}=\boldsymbol{\beta}^{T} \boldsymbol{X}$. The final weights $\beta_{1}^{*}, \beta_{2}^{*}, \ldots, \beta_{n}^{*}$ determine the pre-image with $\boldsymbol{x}^{*}=\sum_{i=1}^{n} \beta_{i}^{*} \boldsymbol{x}_{i}$. From this expression, we can see that in the case of non-negative training data, $\boldsymbol{x}_{1}, \boldsymbol{x}_{2}, \ldots, \boldsymbol{x}_{n} \geq 0$, the resulting pre-image $\boldsymbol{x}^{*}$ will be also non-negative.

By imposing non-negativity of the weights, we get a beneficial side-effect with the sparseness of the solution. This means that a large number of the weights is close to zero, or in other words, only a small number of training data contributes to the final solution. This property is probably due to the non uniqueness of the unconstrained solution, where redundancy in data may result into additive and subtract components that neutralize their contributions. Sparseness is a very desirable property in pattern recognition and machine learning, contributing to a better understanding of the results, in bioinformatics for instance. It is illustrated in the next section on artificial and real datasets.

\section{Experiments}

Three applications of the proposed method are investigated in this section: two applications for data denoising and one on feature extraction. In the first application, two-dimensional artificial data are studied, providing an illustration of the behavior of the algorithm, for two cases: restricting the solution to be non-negative, or forcing the weights to be non-negative, and therefore studying the sparsity of the solution. In the second application, real images from the MNIST database are used to illustrate the efficiency of the proposed method with kernel PCA for denoising. In the third application, we study nonlinear feature 
extraction from real signals. Signals are based on the event-related potentials of the brain activity from the electroencephalograph.

\subsection{Artificial datasets: Denoising scheme}

Let us start with the artificial datasets. For illustration purpose, we consider a two-dimensional space, and apply the denoising scheme separately on three different shapes $^{4}$ : a banana, a donut and a frame. For each example, a set of $n$ samples, given in Figure 3 (upper row), was generated to learn the $m$ eigenvectors. With its quadratic form, we set $m=2$ for the banana dataset, while $m=4$ for the more complicated shape of the frame. Another set of $N$ samples was generated from the same distributions, as given by the (very small) blue dots in Figure 3. Values of the parameters used for each dataset are given in Table 4. It is obvious that these nonlinear shapes cannot be denoised properly using a linear approach, such as the conventional PCA.

First, we compared the non-negative pre-image approach with other unconstrained techniques, including the fixed-point technique defined by (15) and the regularized pre-image estimation [24]. To this end, we considered a setting where all algorithms should give comparable results: all the samples are non-negative. This was done by translating the samples into the positive quadrant, as illustrated in Figure 3 (upper row). For all these algorithms, the noisy version of the data was used at initialization, i.e., $\boldsymbol{x}(t)$ for $t=0$, given by (very small) blue dots in Figure 3. With the number of iterations fixed to $t_{\max }=20$ for iterative algorithms, the denoised samples obtained by these pre-image techniques are represented by red dots. The trajectories obtained at each iteration are represented by green lines (except for the regularized pre-image estimation which is not an iterative technique). As we can see with the length of these lines, the fixed-point algorithm (second row) has slower convergence as opposed to the proposed approach (last row). This is mainly due to the use of the stepsize $\eta$, set here to a fixed value of $\eta=0.3$ for the three datasets. One may take into consideration optimized stepsize values, either with an optimal value for each dataset using a line search technique, or with a stepsize value decreasing at each iteration, i.e., $\eta(t+1)<\eta(t)$. These optimization schema are beyond the scope of this paper. It is worth noting that the results obtained from the regularized pre-image estimation show that one may get into local minima.

We turn now to the approach where the weights are constrained to be non-negative. No restrictions on the data were required in this case, and thus no translation was operated as given above, with samples

\footnotetext{
${ }^{4}$ The banana dataset is defined by a parabola having $\left(x, x^{2}+\xi\right)$ as coordinates, where $x$ on the $x$-axis is uniformly distributed within the interval $[0.5,2.5]$, and $\xi$ is normally distributed with a standard deviation of $\nu=0.2$. The donut dataset is given by data from a circle of radius 0.9 , corrupted by a uniformly distributed noise on $[-\nu, \nu]$, with $\nu=0.4$. The frame dataset is defined by a square of four lines, each of length 2 . The data were uniformly randomly drawn within these lines and corrupted by a uniformly distributed noise on $[-\nu, \nu]$, with $\nu=0.2$.
} 
having positive as well as negative values. We compared three types of kernels: the Gaussian kernel with bandwidth $\sigma=0.7$ as above, the polynomial quadratic kernel with $p=2$ and $c=1$, and the exponential kernel with $\sigma=1$ (see Table 1). For all these kernels, the initial value for $\boldsymbol{\beta}$ given a noisy data $\boldsymbol{x}_{0}$ was set to the solution of $\boldsymbol{x}_{0}=\boldsymbol{\beta}^{T} \boldsymbol{X}$ by retaining only non-negative weights, namely using a pseudo-inverse with

$$
\boldsymbol{\beta}(0)=\left(\boldsymbol{X} \boldsymbol{X}^{T}\right)^{-1} \boldsymbol{X} \boldsymbol{x}_{0}^{T},
$$

for non-negative values; otherwise it was set to zero. Even with only one iteration $(t=1)$ and a small stepsize value of $\eta=0.1$, the proposed algorithm yielded a good denoised pre-image result, as shown in Figure 4 (upper row). Setting the maximum number of iterations to $t=100$, the three kernels gave comparable results reflecting the shape of the banana manifold, as shown in Figure 4 (lower row). This result is in opposition with previous results observed in [23], where Kwok et al. claim that only the Gaussian kernel can be pre-imaged with their work. By constraining the solution to only non-negative weights, as studied in this paper, we see that other kernels provide relevant results.

Now, we turn to the analysis of the model weights, and the sparsity of the solution. To this end, we consider the distribution of the weights $\beta_{1}, \beta_{2}, \ldots, \beta_{n}$ for each of the $N=200$ noisy samples. Figure 5 shows the histogram of such distribution, where each color in the colorbar corresponds to a denoised sample. While we represent here the results of a single iteration, similar results are obtained for larger number of iterations. These results illustrate the fact that the weights are non-negative as expected, lying between 0 and 0.018. Moreover, most of them are close to zero, namely below 0.002 . This is the property of sparsity, well established and often required by a large class of algorithms in machine learning community.

\subsection{Denoising images}

We applied the denoising scheme on real handwritten digits, taken from the MNIST database ${ }^{5}$. From the dataset, we have chosen images of the digit " 0 ". Each image is defined by $28 \times 28$ gray-level pixels, i.e., pixels have values between 0 and 255. Thus, each image can be written as a 784-dimensional vector. The images were corrupted by adding a Salt-and-Pepper noise, with 0.1 density. The images were denoised under the non-negativity of the data, as defined by (16) (see also [14]).

The relevance of the proposed method is now demonstrated for image denoising, and compared to different techniques: the fixed-point iterative method [22], the multi-dimensional scaling method [23], the regularized pre-image estimation [24], and the penalized pre-image [28]. For this purpose, we used the Gaussian kernel

${ }^{5}$ This MNIST database is available at http://yann.lecun.com/exdb/mnist/. 


\footnotetext{
${ }^{6}$ The dataset of ERP recordings are available from the University of Kuopio, Finland and Mika Tarvainen's page http://venda.uku.fi/opiskelu/kurssit/LSA/.
} 
We applied the kernel PCA to extract the first principal axe of these data, in the feature space associated with the Gaussian kernel. The pre-image approach allowed us to go back to the initial space, that is, the signal space. Because signals have negative components ${ }^{7}$, we applied the pre-image technique with the non-negative constraints on the weights. Following some preliminary experimentations, the Gaussian kernel was used, with the bandwidth set to $\sigma=500$, and the stepsize value to the value $\eta=0.1$. Next, we study the influence of the initialization on the algorithm, based on two different initializations.

First, the algorithm was initialized using a random input data, namely $\boldsymbol{x}_{1}$ without loss of generality and shown in Figure 8 (upper figure). Then $\boldsymbol{\beta}(0)=\left(\boldsymbol{X} \boldsymbol{X}^{T}\right)^{-1} \boldsymbol{X} \boldsymbol{x}_{0}^{T}$ for non-negative values, and zero otherwise. Applying the algorithm for $t=100$ iterations gave the feature illustrated in Figure 8 (lower figure). We can easily see both important components of the ERP, the N200 and P300 waves. Moreover, variations of features of interest are opposed to the highly fluctuating initial signal.

In order to study the evolution of the weights at each iteration, we considered the initialization case where all the weights are equal, i.e., $\beta_{k}=1 / n$ for all $k=1,2, \ldots, n$. This corresponds to the average of the data, where the solution results from a uniform contribution of all available data. The evolution of the distribution of these weights over the first five iterations is given in the histograms of Figure 9. This shows that the proposed algorithm resulted into sparse representations, with sparsity increasing at each iteration. The resulting feature is given in Figure 10, which shows both N200 and P300 components, even within the first few iterations. By comparing this technique to the average of some signals in Figure 11 (upper figure) and to all signals in Figure 11 (lower figure), we see that we need all the signals to find the N200 and P300, however, using our method, we only have to use a few signals.

\section{Conclusion and future work}

In this paper, we derived several new theoretical results, and proposed an iterative method to solve the pre-image problem with non-negativity constraints. These constraints were either on the pre-image itself, or on the weights of the model. In this case, we investigated experimentally the sparsity of the representation. Compared to other techniques, simulations showed the effectiveness of the proposed method.

As for future work, we would like to incorporate box constraints, where upper and lower bounds must be satisfied, such as processing gray-level images. We suggest further investigations on other methods that involve pre-image techniques, such as an autoregressive model.

\footnotetext{
${ }^{7}$ To be more precise, measurements of brain activity are always positive. However, practitioners calibrate these measurements, resulting into zero-mean signals.
} 


\section{References}

[1] G. Chen and B. Kégl, "Image denoising with complex ridgelets," Pattern Recognition, vol. 40, no. 2, pp. 578 - 585, 2007.

[2] W.-S. Zheng, J. Lai, S. Liao, and R. He, "Extracting non-negative basis images using pixel dispersion penalty," Pattern Recognition, no. 0, pp. -, 2012.

[3] C. Twining and C. Taylor, "The use of kernel principal component analysis to model data distributions," Pattern Recognition, vol. 36, no. 1, pp. $217-227,2003$.

[4] Z. Yang and E. Oja, "Quadratic nonnegative matrix factorization," Pattern Recognition, vol. 45, no. 4, pp. 1500 - 1510, 2012.

[5] G. Thomas, "A positive optimal deconvolution procedure," IEEE International Conference on Acoustics, Speech, and Signal Processing, ICASSP, vol. 8, pp. 651 - 654, April 1983.

[6] R. Prost and R. Goutte, "Discrete constrained iterative deconvolution algorithms with optimized rate of convergence," Signal Processing, vol. 7, no. 3, pp. 209-230, Dec. 1984.

[7] G. Thomas and N. Souilah, "Utilisation des multiplicateurs de lagrange pour la restauration d'image avec contraintes," Colloques sur le Traitement du Signal et des Images, 1991.

[8] D. Snyder, T. Schulz, and J. O'Sullivan, "Deblurring subject to nonnegativity constraints," IEEE Transactions on Signal Processing, vol. 40, pp. 1143 - 1150, May 1992.

[9] H. Lantéri, M. Roche, O. Cuevas, and C. Aime, "A general method to devise maximum-likelihood signal restoration multiplicative algorithms with non-negativity constraints," Signal Processing, vol. 81, no. 5, pp. 945-974, May 2001.

[10] J. Chen, C. Richard, P. Honeine, H. Snoussi, H. Lantéri, and C. Theys, "Techniques d'apprentissage non-linéaires en ligne avec contraintes de positivite," in Actes de la VI ème Conférence Internationale Francophone d'Automatique, Nancy, France, 2 - 4 Juin 2010.

[11] J. Chen, C. Richard, P. Honeine, H. Lantéri, and C. Theys, "System identification under non-negativity constraints," in Proc. of European Conference on Signal Processing (EUSIPCO). Aalborg, Denmark: Eurasip, 2010.

[12] J. Chen, C. Richard, P. Honeine, and J. C. M. Bermudez, "Non-negative distributed regression for data inference in wireless sensor networks," in Proc. of the 44th Asilomar Conference on Signals, Systems, and Computers, Pacific Grove (CA), USA, 2010.

[13] M. Kallas, P. Honeine, C. Richard, H. Amoud, and C. Francis, "Nonlinear feature extraction using kernel principal component analysis with non-negative pre-image," in Proc. 32nd Annual International Conference of the IEEE Engineering in Medicine and Biology Society (EMBC), Buenos Aires, Argentina, 31 Aug. - 4 Sept. 2010.

[14] M. Kallas, P. Honeine, C. Richard, C. Francis, and H. Amoud, "Non-negative pre-image in machine learning for pattern recognition," in 19th European Signql Processing Conference, Barcelona, Spain, 29 August - 2 September 2011 Submitted.

[15] M. Kallas, H. Amoud, P. Honeine, and C. Francis, "Sur le problème de la pré-image en reconnaissance des formes avec contraintes de non-négativité," in Colloque GRETSI'2011, Bordeaux, France, 5-8 Septembre 2011 Soumis.

[16] H. Han, "Nonnegative principal component analysis for mass spectral serum profiles and biomarker discovery," $B M C$ Bioinformatics, 2010.

[17] R. Zass and A. Shashua, "Nonnegative Sparse PCA," in Neural Information Processing Systems, 2007.

[18] B. Moghaddam, Y. Weiss, and S. Avidan, "Spectral bounds for sparse pca: Exact and greedy algorithms," in Advances in Neural Information Processing Systems. MIT Press, 2006, pp. 915-922.

[19] C. D. Sigg and J. M. Buhmann, "Expectation-maximization for sparse and non-negative pca," in 25th International Conference on Machine Learning (ICML). ACM, 2008.

[20] V. N. Vapnik, Statistical Learning Theory. Wiley-Interscience, September 1998.

[21] B. Schölkopf, A. Smola, and K.-R. Müller, "Nonlinear component analysis as a kernel eigenvalue problem," Neural Comput., vol. 10, pp. 1299-1319, July 1998.

[22] S. Mika, B. Schölkopf, A. Smola, K.-R. Müller, M. Scholz, and G. Rätsch, "Kernel PCA and de-noising in feature spaces," in Proc. of the 1998 conference on advances in neural information processing systems II. Cambridge, MA, USA: MIT Press, 1999, pp. 536-542.

[23] J. T. Kwok and I. W. Tsang, "The pre-image problem in kernel methods," in Proc. of the Twentieth International Conference on Machine Learning, T. Fawcett and N. Mishra, Eds. AAAI Press, 2003, pp. 408-415.

[24] T. J. Abrahamsen and L. K. Hansen, "Regularized pre-image estimation for kernel pca de-noising: Input space regularization and sparse reconstruction," Journal of Signal Processing Systems, vol. 65, no. 3, pp. 403-412, 2011.

[25] P. Honeine and C. Richard, "Solving the pre-image problem in kernel machines: a direct method," in Proc. IEEE Workshop on Machine Learning for Signal Processing (MLSP), Grenoble, France, September 2009.

[26] - "A closed-form solution for the pre-image problem in kernel-based machines," Journal of Signal Processing Systems, vol. 65, pp. 289-299, December 2011.

[27] — "Preimage problem in kernel-based machine learning," IEEE Signal Processing Magazine, vol. 28, no. 2, pp. 77-88, 2011.

[28] W. Zheng, J. Lai, and P. C. Yuen, "Penalized preimage learning in kernel principal component analysis," IEEE Transaction Neural Networks, vol. 21, pp. 551-570, April 2010.

[29] E. J. Candes and M. B. Wakin, "An introduction to compressive sampling," IEEE Signal Processing Magazine, vol. 25, no. 2, pp. 21-30, March 2008.

[30] N. Aronszajn, "Theory of reproducing kernels," Trans. Amer. Math. Soc., vol. 68, pp. 337-404, 1950.

[31] F. Cucker and S. Smale, "On the mathematical foundations of learning," Bulletin of the American Mathematical Society, vol. 39, pp. 1-49, 2002. 
[32] C. J. C. Burges, "Geometry and invariance in kernel based methods," in Advances in kernel methods, B. Schölkopf, C. J. C. Burges, and A. J. Smola, Eds. Cambridge, MA, USA: MIT Press, 1999, pp. 89-116.

[33] B. Schölkopf, A. Smola, and K.-R. Müller, "Nonlinear component analysis as a kernel eigenvalue problem," Neural Comput., vol. 10, no. 5, pp. 1299-1319, 1998.

[34] R. Rosipal, M. Girolami, and L. J. Trejo, "Kernel PCA for feature extraction and de-noising in non-linear regression," Neural Computing and Applications, vol. 10, pp. 231-243, 2000.

[35] G. S. Kimeldorf and G. Wahba, "Some results on Tchebycheffian spline functions," Journal of Mathematical Analysis and Applications, vol. 33, no. 1, pp. 82-95, 1971.

[36] B. Schölkopf, R. Herbrich, and A. J. Smola, "A generalized representer theorem," in Proc. of the 14th Annual Conference on Computational Learning Theory and and 5th European Conference on Computational Learning Theory (COLT '01/EuroCOLT '01). London, UK: Springer-Verlag, 2001, pp. 416-426.

[37] S. D. Georgiadis, "State-space modeling and bayesian methods for evoked potential estimation," Ph.D. dissertation, Department of Applied Physics, University of Kuopio, Finland, May 2007.

[38] M. P. Tarvainen, "Estimation methods for nonstationary biosignals," Ph.D. dissertation, Department of Applied Physics, University of Kuopio, Finland, June 2004. 

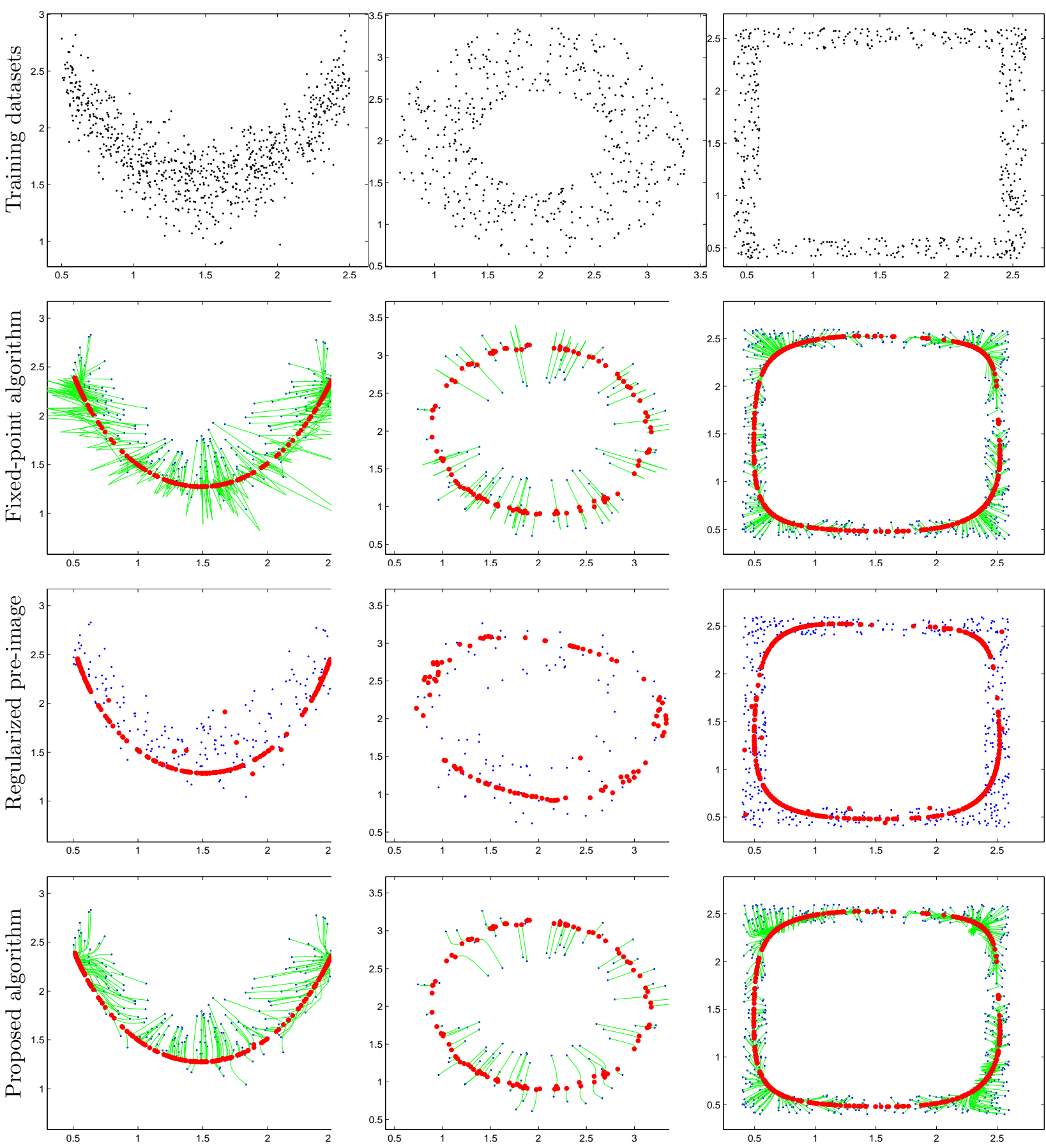

Figure 3: Denoising artificial datasets, for the three shapes: banana (left column), donut (middle column) and frame (right column). A set of training data ( $\nabla$ in upper row) is used for constructing the relevant subspace using the kernel PCA with the Gaussian kernel. Another set of data (designated by •) is denoised (into •) using either the fixed-point (second row), the regularized pre-image estimation method (third row) and the proposed (lower row) algorithms. The evolution of the solution for the iterative methods for the 20 iterations is given with the paths (shown with - ). 


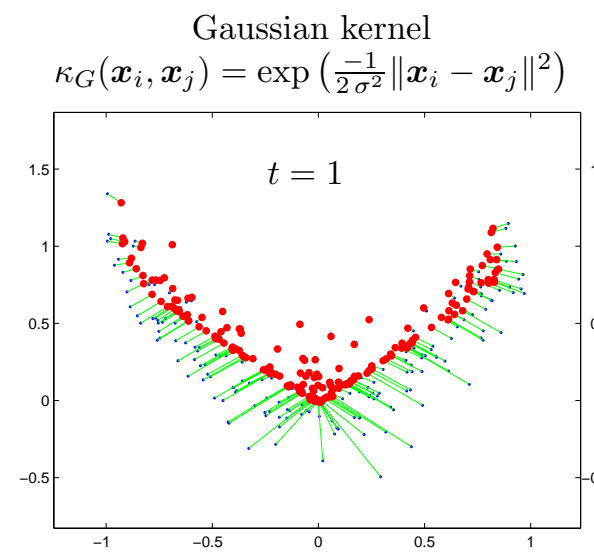

Polynomial kernel

Exponential kernel

$\kappa_{2}\left(\boldsymbol{x}_{i}, \boldsymbol{x}_{j}\right)=\left(1+\boldsymbol{x}_{i} \cdot \boldsymbol{x}_{j}\right)^{2}$ $\kappa_{E}\left(\boldsymbol{x}_{i}, \boldsymbol{x}_{j}\right)=\exp \left(\boldsymbol{x}_{i} \cdot \boldsymbol{x}_{j}\right)$
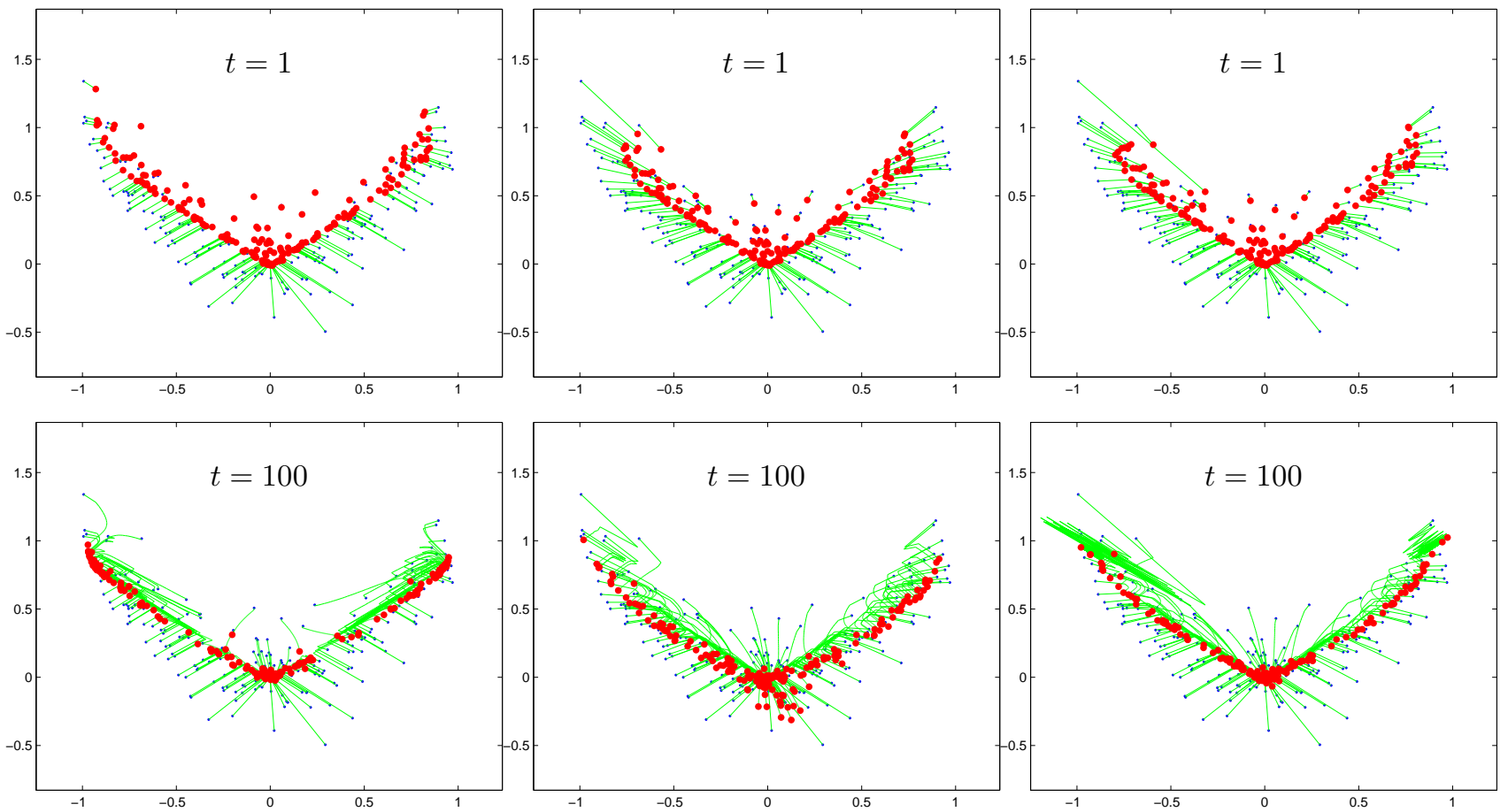

Figure 4: Denoising with constraints on the model weights, of the banana dataset for a single iteration (upper row), and after $t=100$ iterations (lower row). Three different kernels are compared: Gaussian (left column), polynomial (middle column), and exponential (right column) kernels.
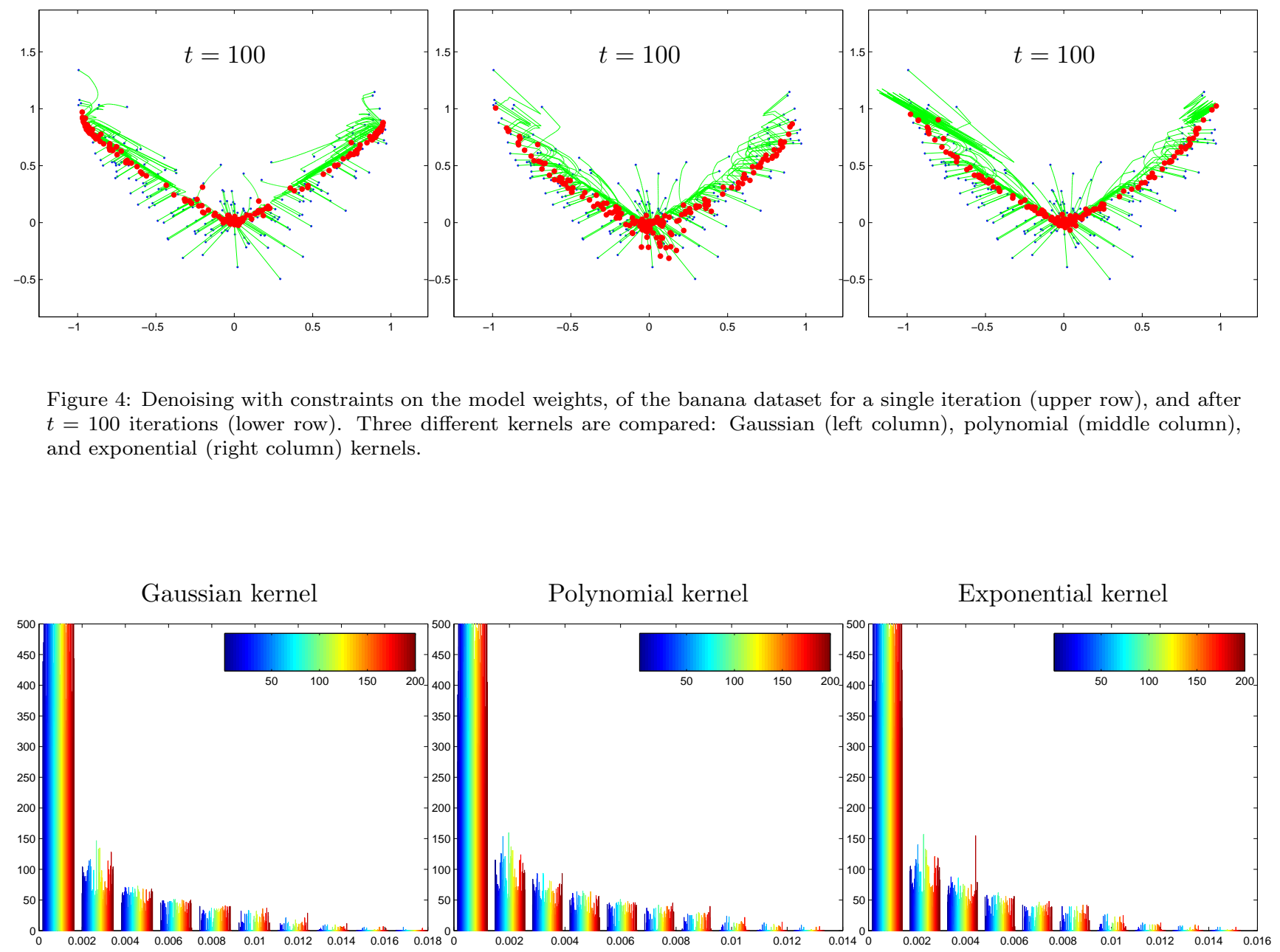

Figure 5: Distribution of the model weights for each of the 200 noisy data from the banana dataset, after only one iteration of our algorithm, corresponding to results given in Figure 4 (upper row). All denoised data (each represented by a color within the colorbar) enjoy the sparsity property, with a large number of weights close to zero. 
Initial images

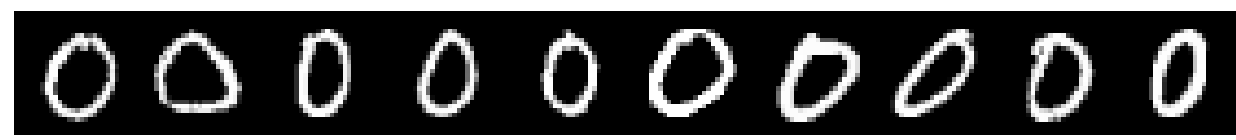

Noisy images

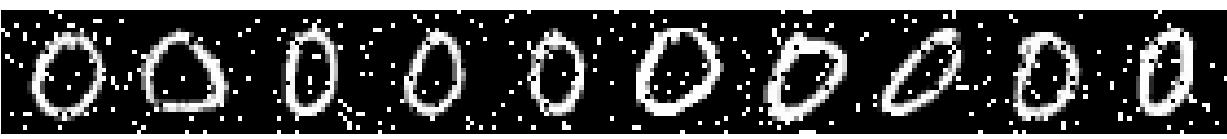

Fixed-point [22]

(100 iterations)

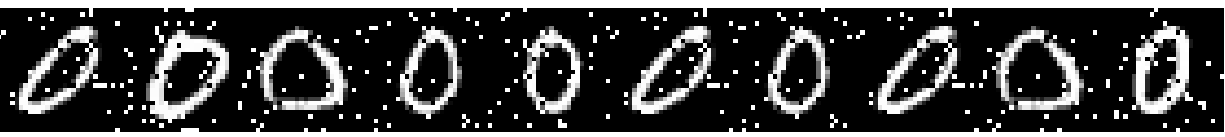

MDS technique [23]

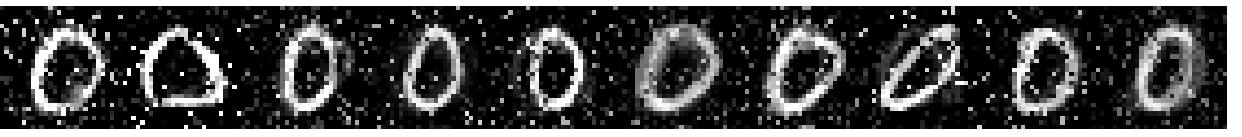

Penalized preimage [28]

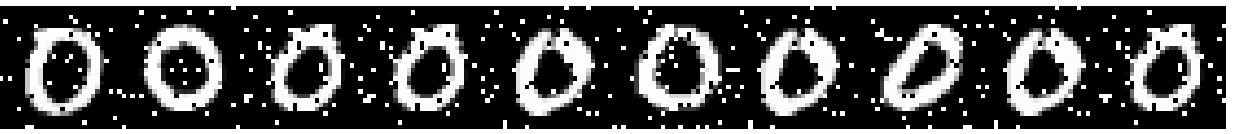

Regularized preimage [24

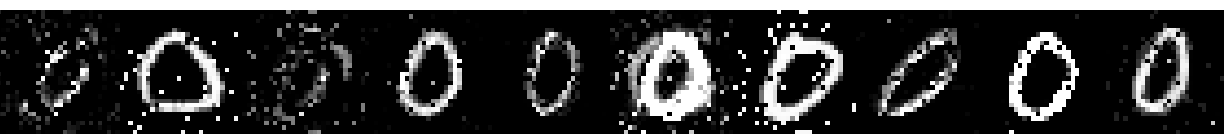

Our method [this paper]

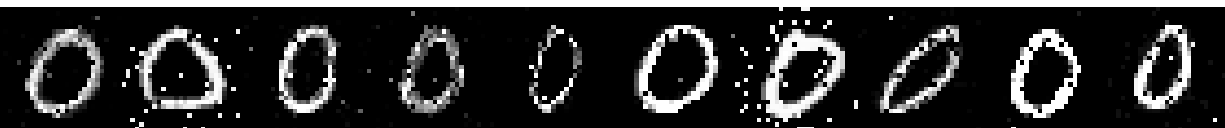

Figure 6: A set of ten "0"-digit images (first row) corrupted by a salt-and-pepper noise of density 0.1 (second row), on which we applied the kernel PCA for data denoising. The pre-image results using the fixed-point iterative algorithm [22] are illustrated (third row), the MDS technique [23] (fourth row), the penalized preimage learning method [28] (fifth row), the regularized preimage estimation technique [24] (sixth row), and the non-negative pre-image with the iterative schema (17) (last row).

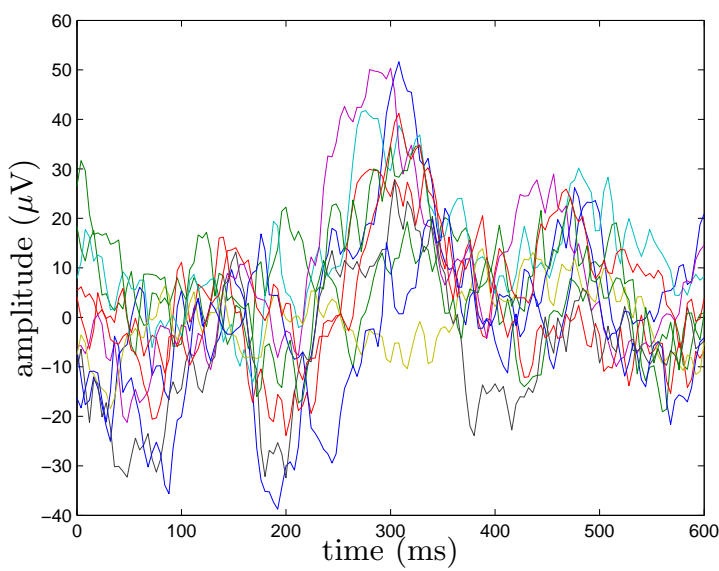

Figure 7: Some ERP signals recorded from the $\mathrm{Cz}$ channel. The diversity of these signals is shown, with some signals not having a positive component around $300 \mathrm{~ms}$ (see for instance - ). 
Initialization signal

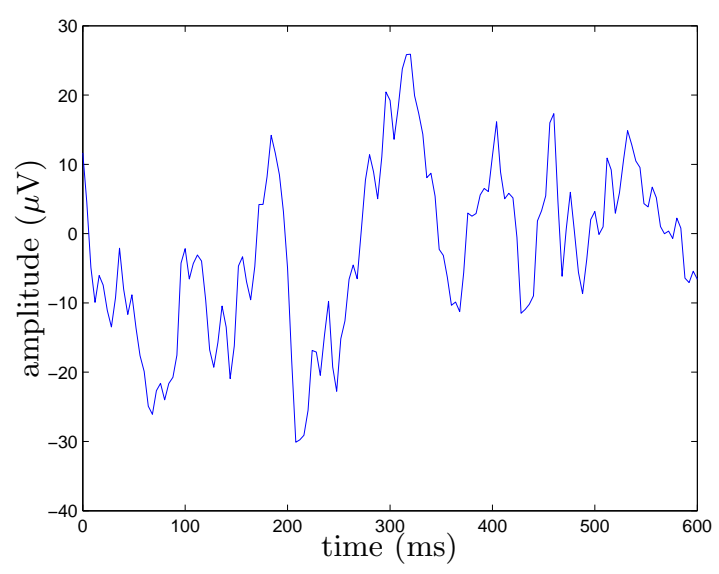

Extracted feature

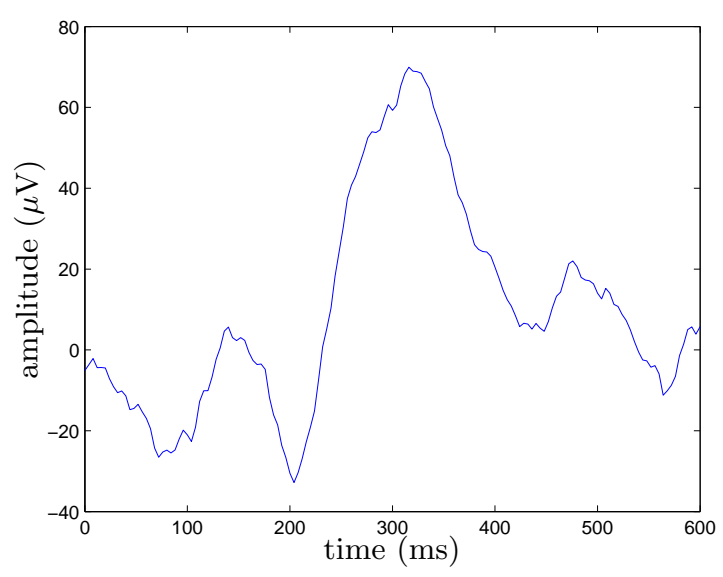

Figure 8: Feature extraction of the ERP data, with the algorithm initialized to the initial signal (upper figure). By pre-imaging the first principal axe of kernel PCA, we get the feature (lower figure). 

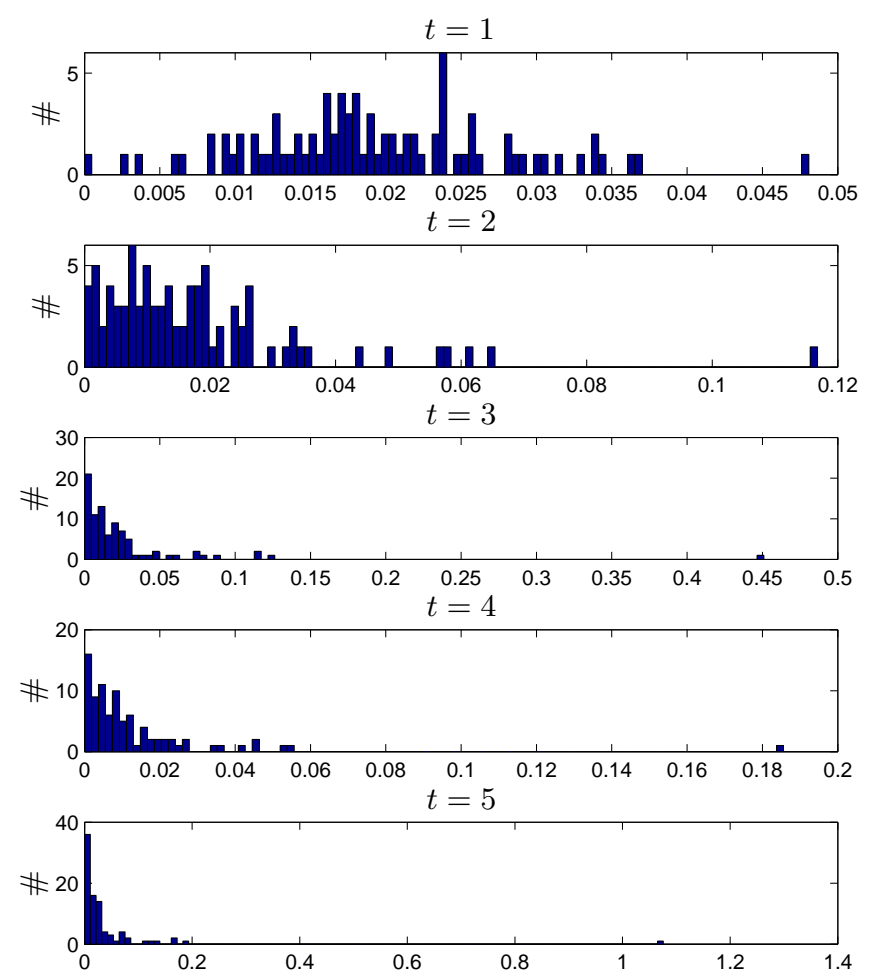

Figure 9: Distribution of the model weights from the first iteration (upper figure) to the fifth iteration (lower figure). This illustrates the evolution of the weights towards a sparse distribution.

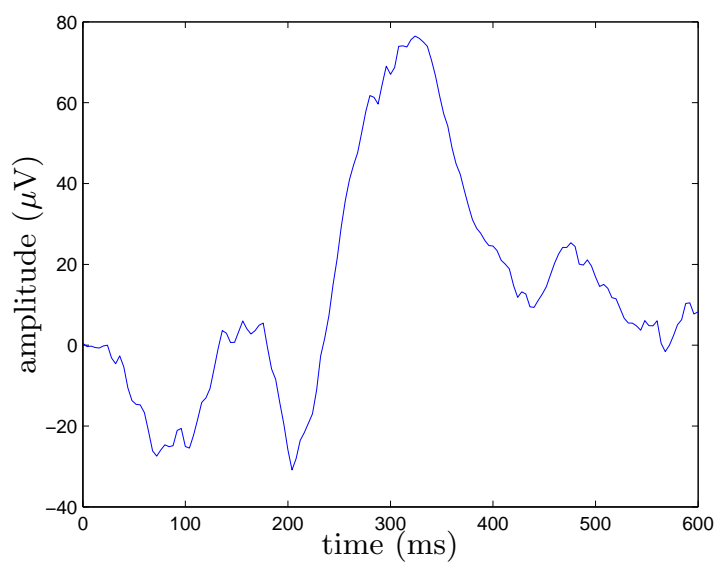

Figure 10: Feature extraction of the ERP data, with the algorithm initialized to the uniform contribution of all available signals, corresponding to $t=5$ in Figure 9 (lower figure). 
Initialization signal

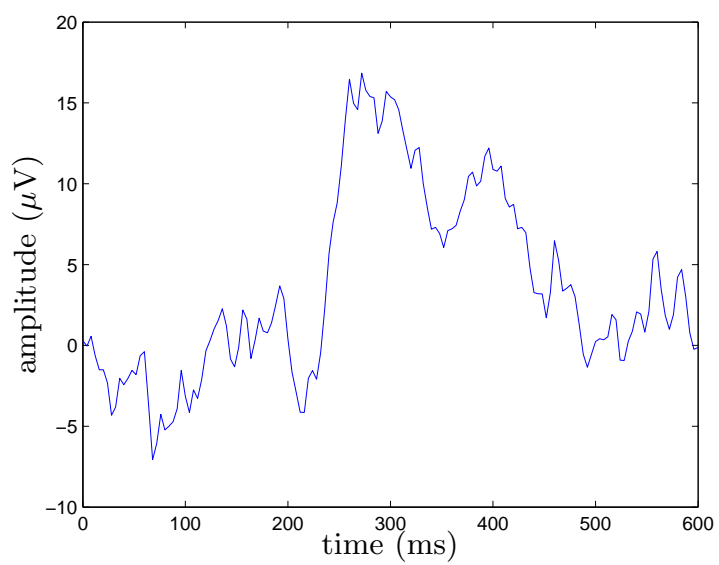

Extracted feature

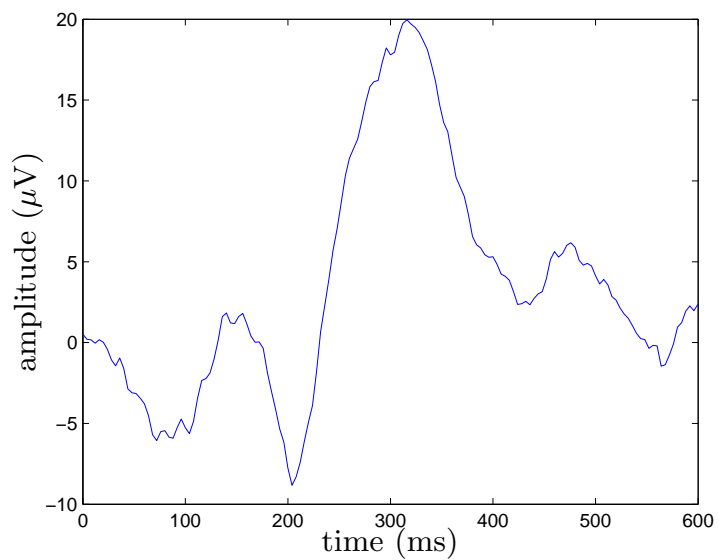

Figure 11: The average of 10 signals (upper figure) and the average of all signals (lower figure) of the ERP data. 